The Astrophysical Journal, 619:570-584, 2005 January 20

(C) 2005. The American Astronomical Society. All rights reserved. Printed in U.S.A.

\title{
FIVE NEW EXTRASOLAR PLANETS ${ }^{1}$
}

\author{
Geoffrey W. Marcy, ${ }^{2}$ R. Paul Butler ${ }^{3}$ Steven S. Vogt, ${ }^{4}$ Debra A. Fischer, ${ }^{2,5}$ \\ Gregory W. Henry, ${ }^{6}$ Greg Laughlin, ${ }^{4}$ Jason T. Wright, ${ }^{2}$ and John A. Johnson ${ }^{2}$ \\ Received 2004 June 25; accepted 2004 September 24
}

\begin{abstract}
We report multiple Doppler measurements of five nearby FGK main-sequence stars and subgiants obtained during the past 4-6 yr at the Keck Observatory. These stars, namely, HD 183263, HD 117207, HD 188015, HD 45350, and HD 99492, all exhibit coherent variations in their Doppler shifts consistent with a planet in Keplerian motion. The five new planets occupy known realms of planetary parameter space, including a wide range of orbital eccentricities, $e=0-0.78$, and semimajor axes, 0.1-3.8 AU, that provide further statistical information about the true distributions of various properties of planetary systems. One of the planets, HD 99492b, has a low minimum mass of $0.112 M_{\text {Jup }}=36 M_{\text {Earth }}$. Four of the five planets orbit beyond $1 \mathrm{AU}$. We describe two quantitative tests of the false alarm probability for Keplerian interpretations of measured velocities. The more robust of these involves Monte Carlo realizations of scrambled velocities as a proxy for noise. Keplerian orbital fits to that "noise" yield the distribution of $\chi_{\nu}^{2}$ to compare with $\chi_{\nu}^{2}$ from the original (unscrambled) velocities. We establish a $1 \%$ false alarm probability as the criterion for candidate planets. All five of these planet-bearing stars are metal-rich, with $[\mathrm{Fe} / \mathrm{H}]>$ +0.27 , reinforcing the strong correlation between planet occurrence and metallicity. From the full sample of 1330 stars monitored at Keck, Lick, and the Anglo-Australian Telescope, the shortest orbital period for any planet is 2.64 days, showing that shorter periods occur less frequently than $0.1 \%$ in the solar neighborhood. Photometric observations were acquired for four of the five host stars with an automatic telescope at Fairborn Observatory. The lack of brightness variations in phase with the radial velocities supports planetary-reflex motion as the cause of the velocity variations. No transits were observed, but their occurrence is not ruled out by our observations.
\end{abstract}

Subject headings: planetary systems - stars: individual (HD 45350, HD 99492, HD 117207, HD 183263, HD 188015)

\section{INTRODUCTION}

To date, $\sim 120$ extrasolar planets are securely known around nearby stars $(d<100 \mathrm{pc})$ all discovered by the Doppler technique, among which are 11 multiplanet systems. ${ }^{7}$ Published descriptions of most of the known planets can be found in the references within and Butler et al. (2002) and Mayor et al. (2004). Most of these planets have minimum masses, $M \sin i$, comparable to or greater than that of Saturn and orbit with semimajor axes of 0.04-6.0 AU. Recently, the first three Neptune-mass planets have been found (Butler et al. 2004; McArthur et al. 2004; Santos et al. 2004b). Two of these Neptunes discovered have remarkably short orbital periods, 2.6 and 2.8 days, consistent with selection effects that limit detection of Neptunes to the closest orbits. No Doppler-detected planets around the nearest 2000 stars have an orbital period of less than 2 days, implying that shorter periods have an occurrence rate of less than 0.0005 .

\footnotetext{
${ }^{1}$ Based on observations obtained at the W. M. Keck Observatory, which is operated jointly by the University of California and the California Institute of Technology. Keck time has been granted by both NASA and the University of California.

2 Department of Astronomy, University of California, Berkeley, CA 947203411; gmarcy@berkeley.edu.

3 Department of Terrestrial Magnetism, Carnegie Institution of Washington, 5241 Broad Branch Road NW, Washington, DC 20015-1305.

${ }_{4}^{4} \mathrm{UCO} /$ Lick Observatory, University of California, Santa Cruz, CA 95064.

5 Department of Physics and Astronomy, San Francisco State University, San Francisco, CA 94132.

${ }^{6}$ Center of Excellence in Information Systems, Tennessee State University, 330 10th Avenue North, Nashville, TN 37203; and Senior Research Associate, Department of Physics and Astronomy, Vanderbilt University, Nashville, TN 37235 .

7 References to published papers and updates on orbital parameters can be found at http://exoplanets.org.
}

The properties of extrasolar planetary systems were recently reviewed by Mayor \& Santos (2003) and Marcy et al. (2003). The planet mass function is consistent with a power law, $d N / d M \propto M^{-1.3}$, with increasing numbers of planets at lower masses (Marcy \& Butler 2000), even after correction for detection efficiency (Jorissen et al. 2001). Our survey of 1330 FGKM main-sequence stars (Marcy et al. 2005; Jones et al. 2005) shows that the "hot" Jupiter-mass planets, orbiting within $0.1 \mathrm{AU}$, exist around $0.8 \% \pm 0.2 \%$ of FGK main-sequence stars. We also find that the number of planets increases with distance from the star from 0.3 to $3 \mathrm{AU}$, in logarithmic bins of orbital distance. Eccentric orbits are distributed nearly uniformly from $e=0$ to 0.7 for planets beyond $0.2 \mathrm{AU}$, and the eccentricity distribution shows no tendency toward circular orbits for the widest orbits. Circular orbits remain rare even for orbital distances beyond $3 \mathrm{AU}$. Systems containing multiple giant planets within $5 \mathrm{AU}$ are found around $1 \%$ of the FGK stars. Remarkably, $20 \%$ of the most metal-rich stars, $[\mathrm{Fe} / \mathrm{H}]>0.3$, harbor planets, while less than $3 \%$ of the metal-poor stars, $[\mathrm{Fe} / \mathrm{H}]<-0.5$, harbor giant planets (Fischer \& Valenti 2005; Santos et al. 2001, 2004a; Reid 2002).

The planetary systems have spawned new theories of the formation, migration, and dynamical interactions of planets (Trilling et al. 2002; Armitage et al. 2002; Chiang \& Murray 2002; Ida $\&$ Lin 2004). Outstanding questions about giant planets include their occurrence as a function of stellar mass (Laws et al. 2003), the origin of orbital eccentricity, the mass function of planets below $1 M_{\text {Sat }}$, and the commonality of circular orbits near and beyond $5 \mathrm{AU}$.

More planets are needed to elucidate their true mass distribution and eccentricity distribution. Here we present five new planets found from our Keck planet survey, offering new 
statistical information about the distribution of orbital eccentricities, semimajor axes, and planetary masses.

\section{TARGET STARS}

We are monitoring the radial velocities of 975 FGKM dwarfs at the Keck 1 telescope, the majority begun in 1997-1998, giving coverage of nearly 6-7 yr. All target stars on our Keck planet search are listed in Wright et al. (2004). The target stars were drawn from the Hipparcos catalog (ESA 1997) with the criteria that they have $B-V>0.55$, have decl. $>-35^{\circ}$, reside less than 3 mag above the main sequence (to avoid photospheric jitter seen in giants), and have no stellar companion within $2^{\prime \prime}$ (to avoid confusion at the entrance slit).

The 975 FGKM stars on the Keck planet search, along with the $\sim 500$ additional stars we monitor at the Lick Observatory and the Anglo Australian Telescope, contain the brightest and closest available FGKM main-sequence and subgiant stars in each of four roughly equal bins of stellar mass from 0.3 to $1.3 M_{\odot}$. The overall survey is magnitude-limited within each mass bin, causing the G stars to be systematically farther than the M dwarfs. Thus, our planet search survey is not volume-limited, except at a given mass on the main sequence. The vast majority of all mainsequence FGKM stars brighter than $V=8$ are included in our survey.

Our spectra include the $\mathrm{Ca}$ II $\mathrm{H}$ and $\mathrm{K}$ emission lines in the near UV, yielding an assessment of chromospheric activity, rotation period, and age (Wright et al. 2004). In brief, we measure the chromospheric emission at the cores of the $\mathrm{Ca}$ II $\mathrm{H}$ and $\mathrm{K}$ lines to construct the ratio of emission to the stellar luminosity, $R_{\mathrm{HK}}^{\prime}$. Representative Keck/HIRES spectra near the $\mathrm{Ca}$ II $\mathrm{H}$ line for all five stars discussed here are shown in Figure 1. The chromospheric emission correlates with stellar age and rotation (Noyes et al. 1984; Wright et al. 2004), and we list $\log R_{\mathrm{HK}}^{\prime}$, $P_{\text {rot }}$, and the inferred age in Table 1 for the five stars presented here. On the basis of $\mathrm{Ca}$ II $\mathrm{H}$ and $\mathrm{K}$ measurements such as these, we retained target stars that are older than $\sim 2$ Gyr, as measured in Wright et al. (2004). However, the discrimination of age among stars older than $2 \mathrm{Gyr}$ is poor (Wright 2004; Pace \& Pasquini 2004).

The original target list included $\sim 200$ additional stars that were later dumped because of a posteriori recognition that they were actually close binaries, giants, or younger than 2 Gyr. Those dumped stars are no longer monitored for radial velocities. In the past 3 years, we added $120 \mathrm{M}$ dwarfs, located within $10 \mathrm{pc}$ and having declination north of $-30^{\circ}$, and all listed in Wright et al. (2004). For the late-type K and M dwarfs, we restricted our selection to stars brighter than $V=11$. Two years ago, we added an additional 100 young FGK stars with ages of 50-500 Myr from the Spitzer Legacy program entitled "Formation and Evolution of Planetary Systems," directed by M. Meyer, L. Hillenbrand, and others, bringing to 975 the total sample of stars that we currently monitor for planets at Keck. The final stellar target list for our planet search is given in Wright et al. (2004), which includes all targets we monitor at both Keck and Lick Observatories.

Our target selection criteria cause several selection effects. The magnitude-limited selection of stars within bins of $B-V$ introduces a Malmquist bias, causing subgiants to be included that reside at larger distances. The subgiants are systematically higher in mass than their main-sequence cohorts of similar $B-V$ because they evolved from the bluer, higher mass region on the main sequence. In this way, our planet search includes stars having mass between 1.2 and $1.5 M_{\odot}$, stars that were otherwise too blue $(B-V<0.55)$ while on the main sequence to be included in the survey.

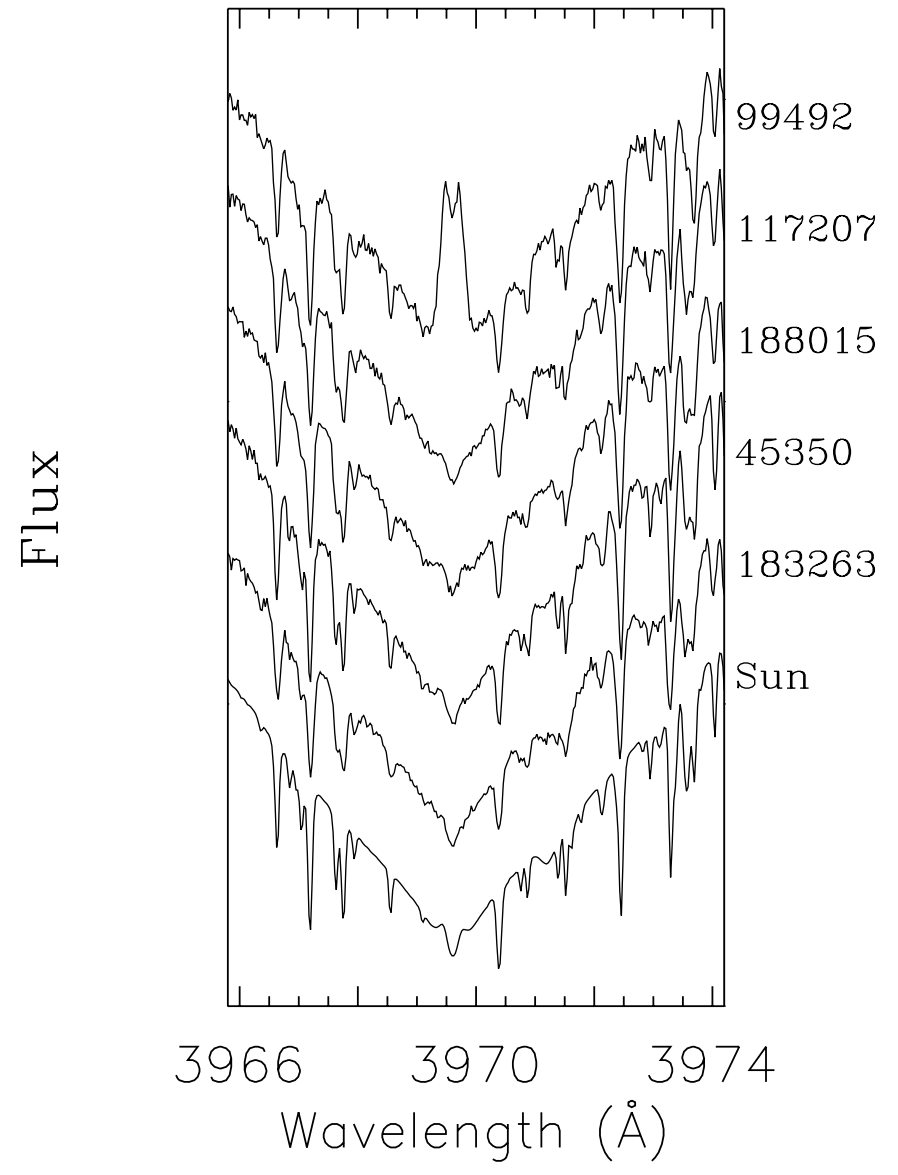

FIG. 1.-Spectra near the $\mathrm{Ca}$ II $\mathrm{H}$ line for all five stars discussed here. The emission reversals reflect magnetic activity on stars that correlates with photospheric velocity jitter (see $\S 4.1$ ). The resulting chromospheric cooling rate, measured as a fraction of stellar luminosity, $\log R_{\mathrm{HK}}^{\prime}$, is listed in Table 1, along with the inferred values of jitter.

A remarkable selection bias stems from the effects of metallicity on the structure and line blanketing of FGK stars near the main sequence (see, for example, Santos et al. 2003). Metalrich stars with $[\mathrm{Fe} / \mathrm{H}]=+0.3$ (roughly $2 \sigma$ above average in the solar neighborhood) are redder by $\sim 0.3 \mathrm{mag}$ in $B-V$ and slightly brighter (Wright 2004; Reid 2002). At a given $B-V$, the stellar population is polluted both by high-mass stars of higher than average metallicity and by low-mass stars of lower than average metallicity. Thus, in a given $B-V$ bin, metallicity correlates positively with stellar mass, despite no physical link between these two stellar properties. Similarly, at a given $B-$ $V$, high-metallicity stars are systematically more luminous than the main sequence of solar metallicity, which in turn are more luminous than the subdwarfs. Thus, in a magnitude-limited sample within a bin of $B-V$, metal-rich stars will be preferentially selected.

This metallicity bias is not the cause of the high occurrence of extrasolar planets found around metal-rich stars. Instead, stars measured a priori as metal-rich have a higher incidence of planets than do stars that are metal-poor (Fischer \& Valenti 2005; Santos et al. 2004b). Roughly $19 \%$ of stars with $[\mathrm{Fe} / \mathrm{H}]>+0.4$ have planets, while less than $3 \%$ of stars with $[\mathrm{Fe} / \mathrm{H}]<-0.3$ harbor detectable planets.

The mass of each target star was determined by linearly interpolating the $M_{V}$ and $(B-V)$ values of each star from Hipparcos (ESA 1997) onto the grid of model isochrones computed by Girardi et al. (2002). No correction was applied to the model 
TABLE 1

Stellar Properties

\begin{tabular}{|c|c|c|c|c|c|c|c|c|c|c|c|c|}
\hline $\begin{array}{l}\text { HD } \\
(1)\end{array}$ & $\begin{array}{l}\text { HIP } \\
(2)\end{array}$ & $\begin{array}{c}\text { Spectral Type } \\
\text { (3) }\end{array}$ & $\begin{array}{c}\pi \\
(\mathrm{mas}) \\
(4)\end{array}$ & $\begin{array}{c}M_{V} \\
(\mathrm{mag}) \\
(5)\end{array}$ & $\begin{array}{c}M_{\text {star }} \\
\left(M_{\odot}\right) \\
(6)\end{array}$ & $\begin{array}{l}\log R_{\mathrm{HK}}^{\prime} \\
\quad(7)\end{array}$ & $\begin{array}{c}\text { Jitter } \\
\left(\mathrm{m} \mathrm{s}^{-1}\right) \\
(8)\end{array}$ & $\begin{array}{c}P_{\text {rot }} \\
\text { (days) } \\
\text { (9) }\end{array}$ & $\begin{array}{c}\log \text { Age } \\
\text { (yr) } \\
(10)\end{array}$ & $\begin{array}{l}T_{\text {eff }} \\
(\mathrm{K}) \\
(11)\end{array}$ & $\begin{array}{c}{[\mathrm{Fe} / \mathrm{H}]} \\
(12)\end{array}$ & $\begin{array}{l}\log g \\
(13)\end{array}$ \\
\hline $117207 \ldots \ldots \ldots \ldots$ & 65808 & G8 IV/V & 30.29 & 4.67 & 1.04 & -5.06 & 3.5 & 36 & 9.84 & 5723 & 0.27 & 4.51 \\
\hline $188015 \ldots \ldots \ldots \ldots$ & 97769 & G5 IV & 19.00 & 4.63 & 1.08 & -5.05 & 4.2 & 36 & 9.82 & 5745 & 0.29 & 4.44 \\
\hline $45350 \ldots \ldots \ldots \ldots \ldots$ & 30860 & G5 V & 20.43 & 4.44 & 1.02 & -5.10 & 3.8 & 39 & 9.90 & 5616 & 0.29 & 4.32 \\
\hline $99492 \ldots \ldots \ldots \ldots . .$. & 55848 & $\mathrm{~K} 2 \mathrm{~V}$ & 55.59 & 6.30 & 0.78 & -4.93 & 2.5 & $45^{\mathrm{a}}$ & $9.6^{\mathrm{a}}$ & 4954 & 0.36 & 4.77 \\
\hline
\end{tabular}

${ }^{\text {a }}$ The values $\log R_{\mathrm{HK}}^{\prime}, P_{\mathrm{rot}}$, and age are poorly determined; these are beyond the domain of $\mathrm{Ca}$ II $\mathrm{H}$ and $\mathrm{K}$ calibration.

grids because we find that the isochrone for solar metallicity stars of age $1 \mathrm{Gyr}$ agrees with the observed positions on the colormagnitude diagram of the Hipparcos main sequence over the $(B-V)$ range spanned by the five target stars. We remain concerned about possible errors of $\sim 5 \%$ due to undiscovered zero-point inadequacies in the Girardi et al. (2002) models, as the bottom of the observed main sequence misses the predicted position from the models by a few tenths of a magnitude.

Girardi et al. (2002) provide model grids for metallicities $[\mathrm{Fe} / \mathrm{H}]=-1.3,-0.7,-0.4,0.0,+0.2,+0.3$, and +0.54 . We used the metallicities from Valenti \& Fischer (2005) to choose the specific model grid for each target star. Stars in each metallicity range that fall below the corresponding model grid's zero-age main sequence, and therefore fall outside of the interpolation range, are interpolated onto the model grid with the next lowest metallicity.

We compared our derived stellar masses to those listed by Allende Prieto \& Lambert (1999), finding our values to be systematically $4 \%$ lower (difference in means) with an rms scatter of $10 \%$. We expect that our stellar masses are probably more accurate because we account for stellar metallicity and use the modern models of Girardi et al. (2002). Nonetheless, we expect the uncertainty of these stellar masses to be $\sim 10 \%$ because of errors of a few percent each in parallax, metallicity, and the models.

Table 1 gives the stellar parameters for the five stars hosting planets that are presented in this paper. Columns (1) and (2) identify the stars with HD and Hipparcos names, respectively. Column (3) gives the spectral type and luminosity class as listed in SIMBAD, column (4) gives the parallax from Hipparcos, and column (5) gives the resulting absolute visual magnitude, $M_{V}$. Column (6) gives the mass of the star, as determined above from the Girardi et al. (2002) models and the metallicities from Valenti \& Fischer (2005) and Fischer \& Valenti (2005). Column (7) gives the chromospheric emission at $\mathrm{Ca}$ II $\mathrm{H}$ and $\mathrm{K}$, namely, $\log R_{\mathrm{HK}}^{\prime}$, and column (8) gives the resulting photospheric jitter in $\mathrm{m} \mathrm{s}^{-1}$, estimated as described in $\S 4.1$. Columns (9) and (10) give the expected rotation and age of the star based on $R_{\mathrm{HK}}^{\prime}$ from Wright et al. (2004) and Noyes et al. (1984).

\section{DOPPLER SHIFT MEASUREMENTS}

For all Doppler measurements, we used our iodine technique (Marcy \& Butler 1992; Butler et al. 1996). The method involves placing iodine molecules at pressure of $0.01 \mathrm{~atm}$ in an absorption cell at a temperature of $50^{\circ} \mathrm{C}$ in front of the focal plane of the Keck I telescope, centered on the optical axis, to superpose iodine lines on the stellar spectrum. The Pyrex iodine cell is sealed so that the column density of iodine remains constant. The iodine lines provide both an indelible wavelength scale and a proxy for the shape of the spectrometer point-spread function (PSF), determined anew for each spectrum and at each wavelength. All time-dependent wavelength scale changes and spectrometer PSF asymmetries are thereby included in the Doppler analysis at each wavelength.

The iodine lines provide wavelength calibration and PSF information on timescales of up to decades to enable secure study of giant planets in orbits of similar size to those in our solar system. Indeed, the Keck iodine cell has not been altered since the beginning of data taking in 1996, preserving the integrity (velocity zero point and scale) of the velocity measurements despite any changes to the optics or detector of the HIRES spectrometer. The spectrometer is operated with a resolution $R \approx 70,000$ and a wavelength range of 3700-6200 $\AA$ (Vogt et al. 1994), although only the region 4950-6000 $\AA$ (with iodine lines) was used in the Doppler analysis. The Doppler shifts from the spectra are determined with the spectral synthesis technique described by (Butler et al. 1996).

For FGKM dwarfs having magnitudes $V=7-11$, the typical exposure times are 3-10 minutes, yielding an $\mathrm{S} / \mathrm{N}$ per pixel in the spectra of $300-75$, respectively. The resulting radial velocity measurements have an internal precision of $2-8 \mathrm{~m} \mathrm{~s}^{-1}$, based on the agreement (uncertainty in the mean) of the $\sim 400$ spectral intervals of $2 \AA$ each.

The vast majority of stars on our survey have Doppler precision of $2.5-4 \mathrm{~m} \mathrm{~s}^{-1}$, depending on rotational $V \sin i$ and absorption line density. Figure 2 shows the Doppler measurements for four representative stars on our program, namely, HD 196850 $(V=6.8, \mathrm{G} 0 \mathrm{~V}), \mathrm{HD} 66171(V=8.2, \mathrm{G} 2 \mathrm{~V}), \mathrm{HD} 199476(V=$ 7.8, G8 V), and HD $202751(V=8.2, \mathrm{~K} 2 \mathrm{~V})$. These four stars exhibit a standard deviation in velocities of between 2.5 and $4 \mathrm{~m}$ $\mathrm{s}^{-1}$, typical of stars on our program as faint as $V=8.2$ with spectral types between F8 and M0. This typical velocity rms stems from several sources, namely, photon-limited errors of $\sim 2 \mathrm{~m} \mathrm{~s}^{-1}$, poor charge transfer properties in the HIRES TEK CCD, and photospheric motions in the star's atmosphere (jitter), as well as any low-mass planets orbiting the star. For the faintest stars, notably M dwarfs with $V=10-11$, the observed Doppler rms for typical stars worsens to 5-7 $\mathrm{m} \mathrm{s}^{-1}$ because of increased photonlimited errors $\left(\sim 5 \mathrm{~m} \mathrm{~s}^{-1}\right)$ and imperfect deconvolution of our stellar templates in the Doppler analysis process.

\section{VELOCITIES AND ORBITAL PARAMETERS FOR FIVE STARS}

\subsection{Keplerian Fits: Errors, Jitter, and Weights}

Velocities for all five stars are presented and analyzed in the following subsections, one for each star. We fit a Keplerian orbit to each set of velocities, using a thorough search of orbital parameter space followed by a Marquardt minimization of $\chi^{2}$. We search for minima near $\sim 40$ prospective periods including those corresponding to the five tallest peaks in the periodogram 


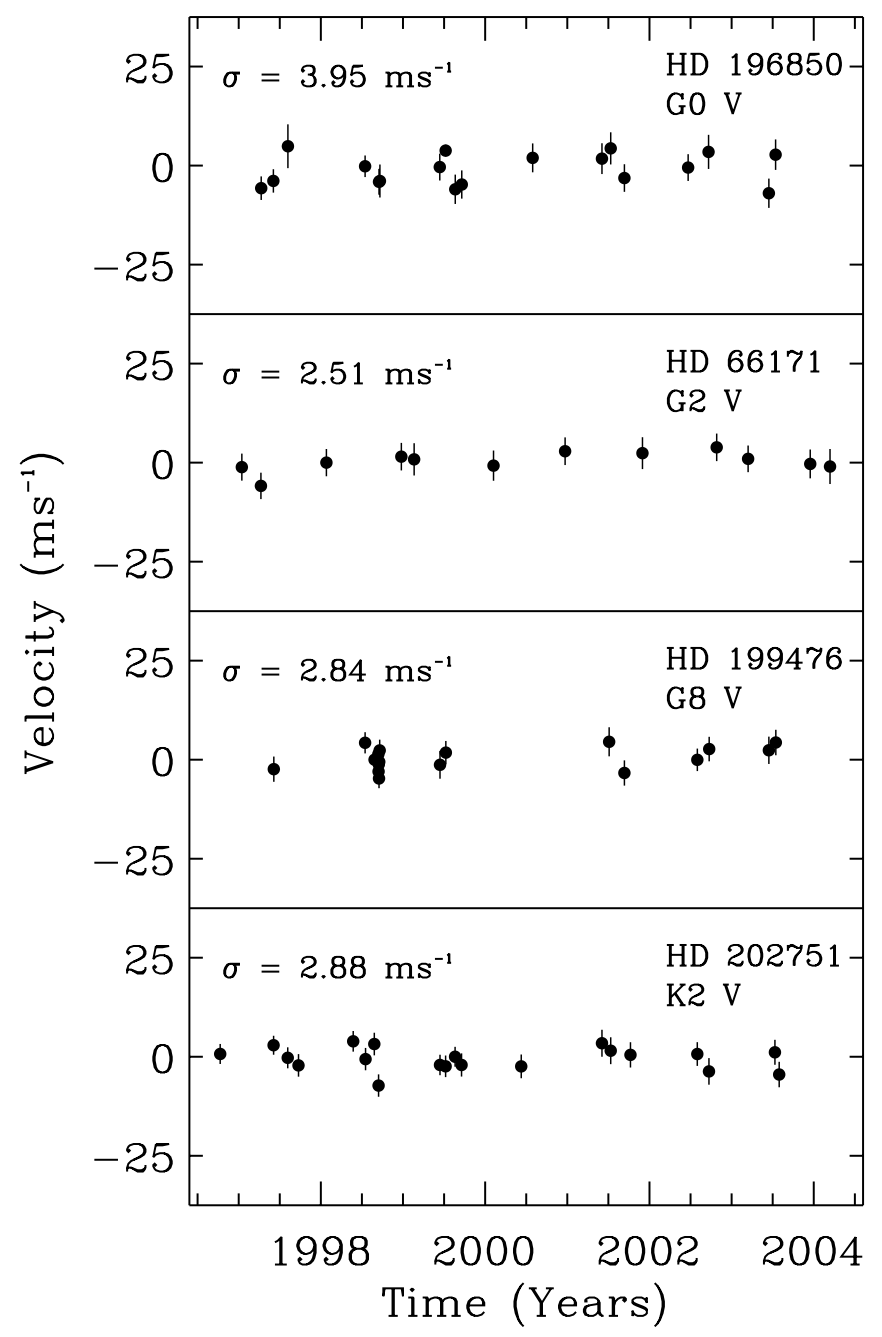

FIG. 2.-Measured velocities vs. time for four representative stars that show no significant velocity variation during 6 years of observations. The standard deviation of velocities ranges from 2.5 to $4.0 \mathrm{~m} \mathrm{~s}^{-1}$, which includes both the errors and photospheric jitter. The four stars, HD 196850, HD 66171, HD 199476, and HD 202751, span the range of spectral types and brightnesses of the candidate planet-bearing stars discussed here, suggesting that they represent empirically the errors and jitter of those stars.

in addition to 35 periods logarithmically spaced between $P=$ 1.0 days and 4 times the duration of observations. The minimization requires that each velocity measurement be assigned a weight that we assemble from two contributions, the internal error and the stellar jitter (Cumming et al. 1999). The internal error is derived from the weighted uncertainty in the mean velocity from the $\sim 400$ chunks, $2 \AA$ long each, of spectra that are independently analyzed for Doppler shift. That internal error, $\epsilon_{i}$, includes photon-limited errors as well as all wavelength-dependent errors that would cause scatter in the resulting velocities among the wavelength chunks.

For each star, we estimate the expected photospheric jitter that will be added in quadrature to each internal measurement error to yield an effective noise for each measurement. The jitter is estimated for each star by associating it with the $\sim 50$ stars that reside near it on the H-R diagram, and finding the best-fit linear relationship between their observed jitter and the chromospheric diagnostic $F_{\mathrm{H}}+F_{\mathrm{K}}$ (Rutten 1984; Cumming et al. 1999; J. T. Wright et al. 2005, in preparation). This jitter assessment is similar to, but based on more stars than, that of Saar et al. (1998) and Santos et al. (2000), the key parameter being the chromospheric emission, $\log R_{\mathrm{HK}}^{\prime}$ (Baliunas et al. 1995).

The resulting jitter values for the 975 target stars are typically between 3 and $10 \mathrm{~m} \mathrm{~s}^{-1}$ with an uncertainty of $\sim 50 \%$, judged from the scatter in the calibration stars. The jitter velocity varies on timescales of minutes, hours, and days because of stellar oscillations, subphotospheric convection, magnetic flux tube evolution, and surface rotation of spots. In contrast, most of our Doppler observations are separated by months. Thus, to a good approximation, the value of jitter for a given observation is drawn independently of the jitter values for other observations of that same star. The total variance in observed velocity can be estimated from the quadrature sum of internal error and jitter. This variance provides the weight, $w_{i}$, for the $i$ th measurement of a star,

$$
w_{i}=1 /\left(\epsilon_{i}^{2}+\text { jitter }^{2}\right)
$$

used in the Marquardt $\chi^{2}$ minimization to fit a Keplerian orbit to a set of velocities. Adding the jitter (a constant for a given star) in quadrature has the effect of softening the differences in the weights among the measured velocities. The resulting bestfit Keplerian orbital parameters for all five stars are given in Table 2.

We determine the uncertainties in the orbital parameters by the following approach. For each star, we find the best-fit Keplerian curve and subtract that from the measured velocities. The resulting residuals are scrambled, keeping the times of observation the same. We then construct 100 realizations of the velocities, each set consisting of the best-fit velocity added to a residual value drawn at random from the scrambled set. In this way we simulate the variation in the individual velocities that stem from both Doppler errors and photospheric jitter, including their actual distributions. For each realization, we determine

TABLE 2

Orbital Parameters

\begin{tabular}{|c|c|c|c|c|c|c|c|c|c|c|}
\hline HD $183263^{\mathrm{a}} \ldots \ldots \ldots \ldots \ldots$ & 634.23 & \pm 5.12 & $2,452,120.61 \pm 6.99$ & $0.38 \pm 0.03$ & $242.0 \pm 5.3$ & $85.0 \pm 2.4$ & 3.69 & 1.52 & 32 & 4.92 \\
\hline HD $188015^{\mathrm{b}} \ldots \ldots \ldots \ldots \ldots$ & 456.46 & \pm 9.12 & $2,451,883.51 \pm 53.37$ & $0.15 \pm 0.09$ & $293.0 \pm 35.7$ & $32.0 \pm 2.4$ & 1.26 & 1.19 & 40 & 7.46 \\
\hline 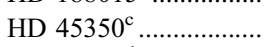 & 890.76 & \pm 37.42 & $2,451,846.48 \pm 24.42$ & $0.78 \pm 0.09$ & $324.1 \pm 7.5$ & $33.0 \pm 4.3$ & 0.98 & 1.77 & 30 & 4.55 \\
\hline 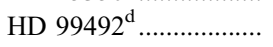 & 17.038 & $8 \pm 0.00536$ & $2,450,462.985 \pm 4.238$ & $0.05 \pm 0.12$ & $89.9 \pm 95.6$ & $10.5 \pm 1.7$ & 0.112 & 0.119 & 41 & 4.27 \\
\hline
\end{tabular}

\footnotetext{
${ }^{\mathrm{a}}$ Linear slope $-32.3 \pm 1.1 \mathrm{~m} \mathrm{~s}^{-1} \mathrm{yr}^{-1}$.

$\mathrm{b}$ Linear slope $-32.3 \pm 1.1 \mathrm{~m} \mathrm{~s}^{-1} \mathrm{yr}^{-1}$.
${ }^{c}$ Linear slope $+3.51 \pm 1.6 \mathrm{~m} \mathrm{~s}^{-1} \mathrm{yr}^{-1}$.

${ }^{c}$ Linear slope $-0.88 \pm 0.64 \mathrm{~m} \mathrm{~s}^{-1} \mathrm{yr}^{-1}$.

$\mathrm{d}$ Linear slope $+1.25 \pm 0.52 \mathrm{~m} \mathrm{~s}^{-1} \mathrm{yr}^{-1}$.
} 
the best-fit Keplerian orbit, accumulating the resulting orbital parameters. We quote the standard deviation of the resulting set of values for each orbital parameter as the " $1 \sigma$ " uncertainty. This approach is likely to underestimate the true uncertainties in the orbital parameters in cases of nonlinear dependence of orbital parameters on the measured velocities. For those cases, a Bayesian Monte Carlo approach to error analysis would be superior, e.g., Ford (2005).

\subsection{HD 183263}

HD 183263 (=HIP 95740) is spectral type G2 IV, with $V=$ $7.86, B-V=0.63$, parallax of 18.93 mas (ESA 1997), and absolute visual magnitude $M_{V}=4.24$, placing it $\sim 0.4$ mag above the average main sequence in the solar neighborhood as computed by Wright (2004). This star is somewhat metal-rich according to Feltzing \& Gustafsson (1998), who find $[\mathrm{Fe} / \mathrm{H}]=$ +0.15 and Valenti \& Fischer (2005), who find $[\mathrm{Fe} / \mathrm{H}]=+0.27$. The high metallicity of HD 183263 partially explains its placement above the main sequence. It is chromospherically quiet, with $S=0.145$ and $\log R_{\mathrm{HK}}^{\prime}=-5.11$, implying a rotation period of 32 days, consistent with an old star (6-10 Gyr) nearing the end of its main-sequence lifetime (Wright et al. 2004; Noyes et al. 1984).

Its mass may be estimated from the Girardi et al. (2002) models, as described in $\S 2$, giving $M=1.17 M_{\odot}$. In comparison, Allende Prieto \& Lambert (1999) find $M=1.10 M_{\odot}$. As our estimate takes account of the metallicity of the star and more modern models, we adopt $M=1.17 \pm 0.1 M_{\odot}$. From our spectra of Ca II $\mathrm{H}$ and $\mathrm{K}$, the expected jitter is $3.8 \mathrm{~m} \mathrm{~s}^{-1}$ (Wright et al. 2004). The stellar parameters for HD 183263 (and all stars in this paper) are listed in Table 1.

Table 3 gives the time of each observation in Julian Dates, the measured radial velocity, and the internal error stemming from the uncertainty in the mean of the 400 spectral chunks. The jitter of $3.8 \mathrm{~m} \mathrm{~s}^{-1}$ is not included in column (3), but is added in quadrature to construct weights in the subsequent Keplerian fit. The velocities are implicitly relative, with no absolute zero point. Figure 3 shows the measured relative velocities for HD 183263, and the associated best-fit Keplerian model. The error bars shown in Figure 3 include both the internal errors and the jitter added in quadrature, but are smaller than the size of the plotted points.

The velocities for HD 183263 in Figure 3 reveal a periodic variation obvious to the eye, requiring no periodogram. However, the best-fit Keplerian has $\sqrt{\chi_{\nu}^{2}}=5.7$, implying that the model does not adequately explain the velocities. However, the inclusion of a linear velocity trend in the model provides a superior fit, giving $\sqrt{\chi_{\nu}^{2}}=0.96$.

The resulting best-fit orbital parameters for the planet are $P=634 \pm 5$ days, $e=0.38$, and $K=85 \mathrm{~m} \mathrm{~s}^{-1}$, implying a minimum mass $M \sin i=3.69 M_{\text {Jup }}$ orbiting with semimajor axis, $a=1.52 \mathrm{AU}$. The linear trend in velocities is $-32 \mathrm{~m} \mathrm{~s}^{-1} \mathrm{yr}^{-1}$ lasting $3 \mathrm{yr}$, implying that a more distant companion orbits the star. The velocity trend allows limits to be associated with the companion: $P>4 \mathrm{yr}, a>2.5 \mathrm{AU}$, and $K>50 \mathrm{~m} \mathrm{~s}^{-1}$, implying a mass constraint $M \sin i>4 M_{\text {Jup }}$. Hipparcos astrometry exhibits no wobble at levels of 1 mas (ESA 1997). As the expected astrometric angular wobble is $\alpha=\left(M_{\text {comp }} / M_{\text {Star }}\right) a / d$, the companion must either have a mass less than $50 M_{\text {Jup }}$, or it must orbit with $P>8 \mathrm{yr}$, so that the wobble is absorbed into the propermotion solution of the Hipparcos solution. As our spectra reveal no evidence of a second set of absorption lines at the $1 \%$ flux level, this companion is likely substellar and possibly a second planet in the system.
TABLE 3

Radial Velocities for HD 183263

\begin{tabular}{|c|c|c|}
\hline $\begin{array}{c}\mathrm{JD}-2,440,000 \\
\text { (1) }\end{array}$ & $\begin{array}{l}\text { Radial Velocity } \\
\left(\mathrm{m} \mathrm{s}^{-1}\right) \\
(2)\end{array}$ & $\begin{array}{c}\text { Uncertainty } \\
\left(\mathrm{m} \mathrm{s}^{-1}\right) \\
\text { (3) }\end{array}$ \\
\hline $12,094.892 \ldots \ldots \ldots \ldots \ldots \ldots \ldots \ldots \ldots$ & -51.79 & 4.0 \\
\hline $12,098.019 \ldots \ldots \ldots \ldots \ldots \ldots \ldots \ldots \ldots \ldots$ & -50.15 & 4.3 \\
\hline $12,099.042 \ldots \ldots \ldots \ldots \ldots \ldots \ldots \ldots \ldots \ldots$ & -54.55 & 4.1 \\
\hline 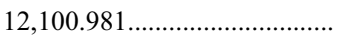 & -54.70 & 4.4 \\
\hline 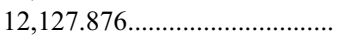 & -10.23 & 4.1 \\
\hline 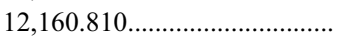 & 42.04 & 4.4 \\
\hline 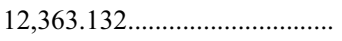 & 58.81 & 4.4 \\
\hline 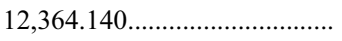 & 58.42 & 4.3 \\
\hline $12,390.121 \ldots \ldots \ldots \ldots \ldots \ldots \ldots \ldots \ldots$ & 47.28 & 4.2 \\
\hline $12,445.964 \ldots \ldots \ldots \ldots \ldots \ldots \ldots \ldots \ldots$ & 21.18 & 4.5 \\
\hline $12,486.802 \ldots \ldots \ldots \ldots \ldots \ldots \ldots \ldots \ldots$ & 6.16 & 3.9 \\
\hline $12,535.759 \ldots \ldots \ldots \ldots \ldots \ldots \ldots \ldots$ & -14.64 & 4.0 \\
\hline $12,572.698 \ldots \ldots \ldots \ldots \ldots \ldots \ldots \ldots \ldots$ & -51.77 & 6.5 \\
\hline 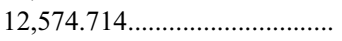 & -49.11 & 6.6 \\
\hline 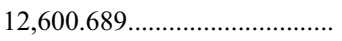 & -59.80 & 4.2 \\
\hline 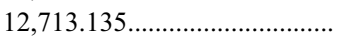 & -126.77 & 3.5 \\
\hline $12,778.086 \ldots \ldots \ldots \ldots \ldots \ldots \ldots \ldots \ldots \ldots$ & -35.29 & 4.0 \\
\hline $12,804.114 \ldots \ldots \ldots \ldots \ldots \ldots \ldots \ldots \ldots$ & 0.00 & 4.0 \\
\hline $12,832.827 \ldots \ldots \ldots \ldots \ldots \ldots \ldots \ldots \ldots$ & 25.47 & 4.2 \\
\hline $12,848.907 \ldots \ldots \ldots \ldots \ldots \ldots \ldots \ldots \ldots \ldots$ & 27.12 & 4.9 \\
\hline $12,855.981 \ldots \ldots \ldots$ & 39.31 & 4.2 \\
\hline 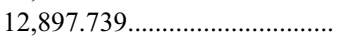 & 34.93 & 4.0 \\
\hline $12,924.737 \ldots \ldots \ldots$ & 21.73 & 6.4 \\
\hline $12,987.696 \ldots \ldots \ldots$ & -2.25 & 6.1 \\
\hline $12,988.701 \ldots \ldots \ldots \ldots \ldots \ldots \ldots \ldots \ldots$ & 7.75 & 2.8 \\
\hline $12,989.700 \ldots \ldots \ldots \ldots \ldots \ldots \ldots \ldots \ldots \ldots$ & 4.89 & 3.6 \\
\hline 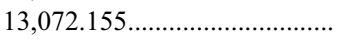 & -37.49 & 3.7 \\
\hline 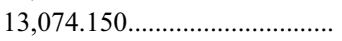 & -32.79 & 4.2 \\
\hline $13,077.138 \ldots \ldots \ldots \ldots \ldots \ldots$ & -25.80 & 3.9 \\
\hline
\end{tabular}

\subsection{HD 117207}

HD 117207 (=HIP 65808) is spectral type G8 IV/V, with $V=$ 7.26, $B-V=0.72$, parallax of 30.29 mas (ESA 1997), and absolute visual magnitude $M_{V}=4.67$, placing it $\sim 0.55 \mathrm{mag}$ above the main sequence. It is chromospherically quiet, with $S=0.155$ and $\log R_{\mathrm{HK}}^{\prime}=-5.06$ (Wright et al. 2004) consistent with Henry et al. (1996), who find $\log R_{\mathrm{HK}}^{\prime}=-5.00$. The implied rotation period is 36 days from Noyes et al. (1984). Valenti \& Fischer (2005) find $[\mathrm{Fe} / \mathrm{H}]=+0.27$ from LTE fitting of multiple lines in our spectra. Its placement above the main sequence and low chromospheric activity are consistent with an old, quiescent star of age 6-10 Gyr.

We estimated the stellar mass from the Girardi et al. (2002) models, as described in $\S 2$, giving $M=1.04 M_{\odot}$, in agreement with Allende Prieto \& Lambert (1999), who find $M=1.03 M_{\odot}$. As our estimate takes account of the metallicity of the star, we adopt $M=1.04 \pm 0.1 M_{\odot}$. From our spectra of $\mathrm{Ca}$ II $\mathrm{H}$ and $\mathrm{K}$, the expected jitter is $3.56 \mathrm{~m} \mathrm{~s}^{-1}$ (J. T. Wright et al. 2005, in preparation). The stellar parameters are listed in Table 1.

Table 4 gives the time of each observation in Julian Dates, the measured (relative) radial velocity, and the uncertainty. The expected jitter of $3.56 \mathrm{~m} \mathrm{~s}^{-1}$ is not included in the table, but is added to the errors in quadrature in the Keplerian fit. Figure 4 shows the measured velocities for HD 117207 and the associated best-fit Keplerian model.

The velocities for HD 117207 in Figure 4 reveal an apparently complete orbit. More observations in the next 2 years will help confirm the periodicity. The best-fit Keplerian has $\sqrt{\chi_{\nu}^{2}}=$ 1.00 , implying that the Keplerian model adequately explains the 


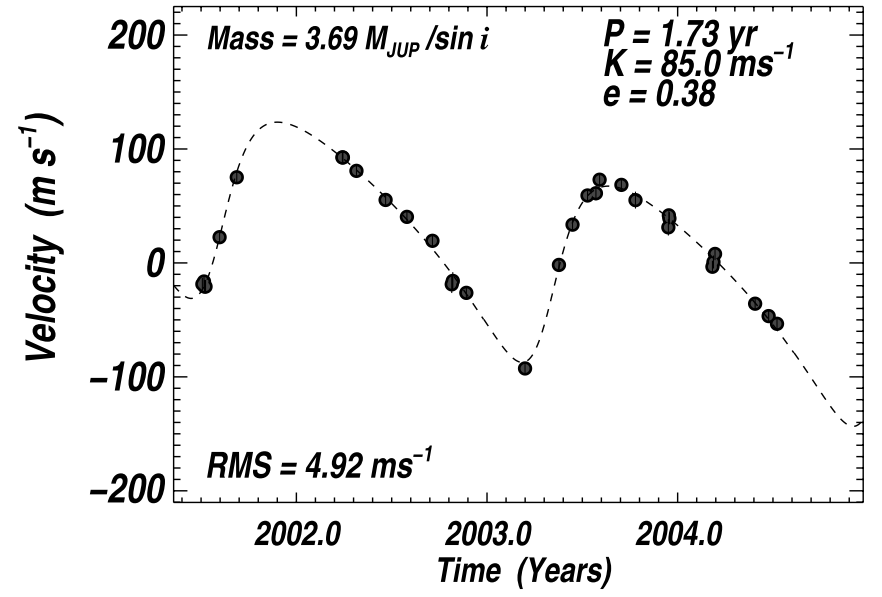

FIG. 3.-Measured velocity vs. time for HD 183263 (dots) with the associated best-fit Keplerian with period $P=633$ days and $M \sin i=3.75 M_{\text {Jup }}$. A linear trend of $33 \mathrm{~m} \mathrm{~s}^{-1} \mathrm{yr}^{-1}$ is apparent, indicating a more distant companion, likely substellar. Error bars are smaller than the plotted points.

TABLE 4

Radial Velocities for HD 117207

\begin{tabular}{|c|c|c|}
\hline $\mathrm{JD}-2,440,000$ & $\begin{array}{l}\text { Radial Velocity } \\
\qquad\left(\mathrm{m} \mathrm{s}^{-1}\right)\end{array}$ & $\begin{array}{c}\text { Uncertainty } \\
\left(\mathrm{m} \mathrm{s}^{-1}\right)\end{array}$ \\
\hline $10,463.162 \ldots \ldots \ldots \ldots \ldots \ldots \ldots \ldots$ & 19.0318 & 3.1 \\
\hline $10,546.977 \ldots \ldots \ldots \ldots \ldots \ldots \ldots$ & 5.82358 & 2.7 \\
\hline $10,838.124 \ldots \ldots \ldots \ldots \ldots \ldots \ldots$ & -9.81886 & 2.7 \\
\hline 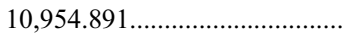 & -28.2798 & 2.7 \\
\hline $10,955.851 \ldots \ldots \ldots \ldots \ldots \ldots \ldots \ldots . .$. & -29.0574 & 2.8 \\
\hline $11,227.056 \ldots \ldots \ldots \ldots \ldots \ldots \ldots \ldots \ldots \ldots \ldots \ldots$ & -30.6795 & 2.9 \\
\hline 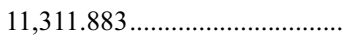 & -42.9408 & 8.2 \\
\hline 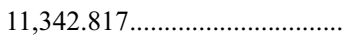 & -32.0488 & 2.7 \\
\hline 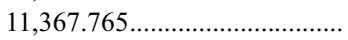 & -35.0539 & 2.3 \\
\hline $11,371.749 \ldots \ldots \ldots \ldots \ldots \ldots \ldots \ldots \ldots \ldots \ldots \ldots$ & -32.8409 & 2.8 \\
\hline $11,372.752 \ldots \ldots \ldots \ldots \ldots \ldots \ldots \ldots \ldots \ldots \ldots \ldots \ldots$ & -33.4615 & 2.7 \\
\hline 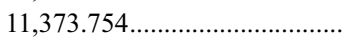 & -39.5277 & 3.0 \\
\hline $11,582.050 \ldots \ldots \ldots \ldots \ldots \ldots \ldots \ldots \ldots \ldots$ & -19.9703 & 3.6 \\
\hline $11,679.882 \ldots \ldots \ldots \ldots \ldots \ldots \ldots \ldots \ldots \ldots$ & -22.6980 & 2.9 \\
\hline $11,702.883 \ldots \ldots \ldots \ldots \ldots \ldots \ldots \ldots \ldots \ldots \ldots$ & -26.0960 & 2.7 \\
\hline 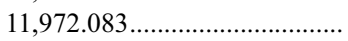 & -9.16290 & 3.3 \\
\hline 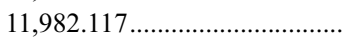 & -9.58160 & 2.9 \\
\hline 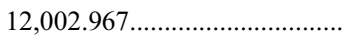 & 0.00000 & 3.5 \\
\hline 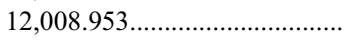 & -6.13087 & 3.3 \\
\hline 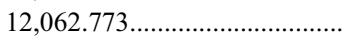 & -6.79053 & 3.0 \\
\hline 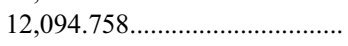 & -6.95602 & 3.0 \\
\hline $12,100.765 \ldots \ldots \ldots \ldots \ldots \ldots \ldots \ldots \ldots \ldots \ldots$ & -3.43360 & 3.3 \\
\hline 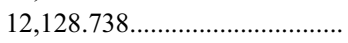 & -5.66888 & 3.6 \\
\hline $12,334.092 \ldots \ldots \ldots \ldots \ldots \ldots \ldots \ldots \ldots \ldots \ldots \ldots$ & 8.65721 & 3.6 \\
\hline 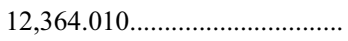 & 17.3127 & 2.3 \\
\hline 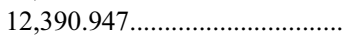 & 4.55621 & 3.1 \\
\hline 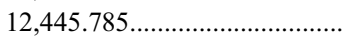 & 11.8581 & 3.0 \\
\hline $12,681.156 \ldots \ldots \ldots \ldots \ldots \ldots \ldots \ldots \ldots$ & 22.4985 & 3.2 \\
\hline $12,712.025 \ldots \ldots \ldots \ldots \ldots \ldots \ldots \ldots \ldots \ldots \ldots \ldots \ldots . .$. & 21.4198 & 3.2 \\
\hline 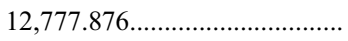 & 26.7895 & 3.1 \\
\hline 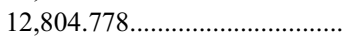 & 40.0828 & 3.6 \\
\hline $12,850.740 \ldots \ldots \ldots \ldots \ldots \ldots \ldots \ldots \ldots \ldots \ldots \ldots$ & 19.0017 & 2.7 \\
\hline 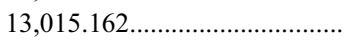 & 23.8500 & 2.8 \\
\hline 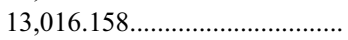 & 21.8146 & 3.0 \\
\hline 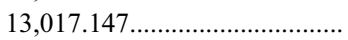 & 23.4381 & 2.9 \\
\hline
\end{tabular}

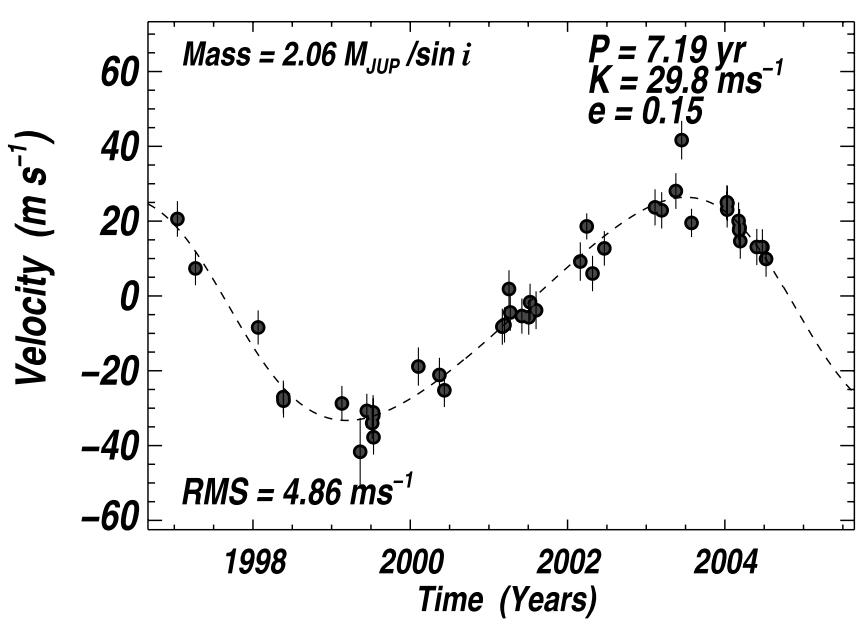

FIG. 4.-Measured velocity vs. time for HD 117207 (dots) with the associated best-fit Keplerian with period $P=7.19 \mathrm{yr}, e=0.16$, and $M \sin i=2.06 M_{\mathrm{Jup}}$.

velocities. The resulting best-fit orbital parameters for the planet are $P=7.19 \pm 0.3 \mathrm{yr}, e=0.16$, and $K=29.8 \mathrm{~m} \mathrm{~s}^{-1}$, implying a minimum mass $M \sin i=2.06 M_{\text {Jup }}$ orbiting with semimajor axis $a=3.78$ AU.

With only one orbit in hand, the constraints on the orbital parameters are modest. We carried out a Monte Carlo test in which artificial Gaussian noise, equal to our actual errors and jitter, were added to the best-fit Keplerian orbit, evaluated at our actual times of observation. We ran 100 trials, redetermining the best-fit Keplerian orbit for each trial. The standard deviation of the resulting orbital parameters is deemed a measure of their uncertainty. We find that the orbital period carries an uncertainty of 61 days, the eccentricity is uncertain by 0.08 , and the velocity amplitude is uncertain by $1.6 \mathrm{~m} \mathrm{~s}^{-1}$. Thus, the orbit is quite well constrained at the $10 \%$ level.

\section{4. $H D 188015$}

HD 188015 (=HIP 97769) is spectral type G5 IV, with $V=$ $8.24, B-V=0.73$, parallax of 19.00 mas (ESA 1997), and absolute visual magnitude $M_{V}=4.63$, placing it $\sim 0.5$ mag above the main sequence. It is chromospherically quiet, with $S=0.15$ and $\log R_{\mathrm{HK}}^{\prime}=-5.05$ (Wright et al. 2004). This star was suggested for inclusion in our sample by Laughlin (2000), who finds it to be metal-rich, $[\mathrm{Fe} / \mathrm{H}]=+0.29$, based on calibration of narrowband photometry, in agreement with Valenti \& Fischer (2005), who carry out LTE fitting of our spectra giving $[\mathrm{Fe} / \mathrm{H}]=+0.23$. The implied rotation period is 36 days. Its placement above the main sequence and low chromospheric activity are consistent with an old, quiescent star (6-10 Gyr).

We estimated the stellar mass from the Girardi et al. (2002) models, as described in $\S 2$, giving $M=1.08 M_{\odot}$. In comparison, Allende Prieto \& Lambert (1999) find $M=1.03 M_{\odot}$. As our estimate takes account of the metallicity of the star, we adopt $M=$ $1.08 \pm 0.1 M_{\odot}$. From our spectra of $\mathrm{Ca}$ II $\mathrm{H}$ and $\mathrm{K}$, the expected jitter is $4.22 \mathrm{~m} \mathrm{~s}^{-1}$. Stellar parameters are listed in Table 1 .

Table 5 gives the time of observation, the measured radial velocity, and the internal error. Figure 5 shows the measured relative velocities for HD 188015 and the associated best-fit Keplerian model. The error bars shown in Figure 5 include both the internal errors and jitter, added in quadrature.

The velocities for HD 188015 in Figure 5 reveal an obvious periodicity, with a period somewhat over a year and a semiamplitude of roughly $40 \mathrm{~m} \mathrm{~s}^{-1}$ apparent to the eye. The bestfit simple Keplerian has a $\sqrt{\chi_{\nu}^{2}}=1.36$ and residuals with 
TABLE 5

Radial Velocities for HD 188015

\begin{tabular}{|c|c|c|}
\hline $\mathrm{JD}-2,440,000$ & $\begin{array}{l}\text { Radial Velocity } \\
\qquad\left(\mathrm{m} \mathrm{s}^{-1}\right)\end{array}$ & $\begin{array}{l}\text { Uncertainty } \\
\left(\mathrm{m} \mathrm{s}^{-1}\right)\end{array}$ \\
\hline $11,754.896 \ldots .$. & -66.5932 & 5.0 \\
\hline $11,792.767 \ldots \ldots \ldots \ldots \ldots \ldots \ldots \ldots \ldots \ldots \ldots \ldots \ldots$ & -52.2866 & 4.1 \\
\hline 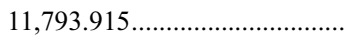 & -38.7390 & 4.0 \\
\hline $12,062.027 \ldots \ldots \ldots \ldots \ldots \ldots \ldots \ldots \ldots \ldots \ldots \ldots$ & -25.3485 & 7.6 \\
\hline 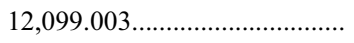 & -13.1770 & 3.8 \\
\hline 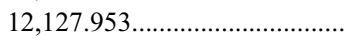 & -49.7930 & 4.3 \\
\hline $12,132.990 \ldots \ldots \ldots \ldots \ldots \ldots \ldots \ldots$ & -24.7541 & 3.9 \\
\hline $12,133.755 \ldots \ldots \ldots \ldots \ldots \ldots \ldots \ldots \ldots$ & -34.8433 & 4.3 \\
\hline $12,447.001 \ldots \ldots \ldots \ldots \ldots \ldots \ldots \ldots \ldots$ & 5.39621 & 4.2 \\
\hline $12,536.854 \ldots \ldots \ldots \ldots \ldots \ldots \ldots \ldots$ & -9.94483 & 4.4 \\
\hline 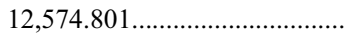 & -26.2157 & 4.6 \\
\hline 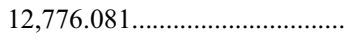 & -10.8480 & 4.1 \\
\hline $12,778.129 \ldots \ldots \ldots \ldots \ldots \ldots \ldots \ldots \ldots$ & -6.72055 & 4.1 \\
\hline $12,803.991 \ldots \ldots \ldots \ldots \ldots \ldots \ldots \ldots \ldots$ & 3.41108 & 3.5 \\
\hline $12,805.078 \ldots \ldots \ldots \ldots \ldots \ldots \ldots \ldots \ldots \ldots \ldots \ldots \ldots \ldots \ldots$ & 7.06809 & 3.3 \\
\hline 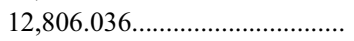 & 3.85333 & 3.7 \\
\hline $12,806.946 \ldots \ldots \ldots \ldots \ldots \ldots \ldots \ldots$ & 0.00000 & 3.6 \\
\hline $12,828.928 \ldots \ldots \ldots \ldots \ldots \ldots \ldots \ldots \ldots$ & 19.0491 & 3.7 \\
\hline $12,832.879 \ldots \ldots \ldots \ldots \ldots \ldots \ldots \ldots \ldots \ldots$ & 14.8929 & 3.7 \\
\hline $12,833.897 \ldots \ldots \ldots \ldots \ldots \ldots \ldots \ldots \ldots \ldots$ & 19.4294 & 4.1 \\
\hline $12,834.831 \ldots \ldots \ldots \ldots \ldots \ldots \ldots \ldots \ldots \ldots \ldots \ldots \ldots \ldots$ & 18.6605 & 3.9 \\
\hline $12,835.955 \ldots \ldots \ldots \ldots \ldots \ldots \ldots \ldots \ldots \ldots \ldots \ldots \ldots \ldots$ & 22.2396 & 3.9 \\
\hline 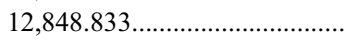 & 18.5657 & 3.9 \\
\hline $12,850.925 \ldots \ldots \ldots \ldots \ldots \ldots \ldots \ldots \ldots$ & 16.7080 & 3.3 \\
\hline $12,853.962 \ldots \ldots \ldots \ldots \ldots \ldots \ldots \ldots \ldots$ & 30.7468 & 3.9 \\
\hline $12,854.992 \ldots \ldots \ldots \ldots \ldots \ldots \ldots \ldots \ldots \ldots \ldots \ldots \ldots \ldots$ & 30.7676 & 3.8 \\
\hline $12,855.985 \ldots \ldots \ldots \ldots \ldots \ldots \ldots \ldots \ldots \ldots \ldots \ldots \ldots$ & 29.6136 & 3.8 \\
\hline $12,897.807 \ldots \ldots \ldots \ldots \ldots \ldots \ldots \ldots \ldots \ldots$ & 14.1409 & 3.8 \\
\hline $12,898.779 \ldots \ldots \ldots \ldots \ldots \ldots \ldots \ldots \ldots \ldots \ldots \ldots$ & 13.1737 & 3.9 \\
\hline $12,924.717 \ldots \ldots \ldots \ldots \ldots \ldots \ldots \ldots . .$. & 9.24128 & 3.8 \\
\hline $13,014.701 \ldots \ldots \ldots \ldots \ldots \ldots \ldots \ldots \ldots$ & -5.17810 & 4.4 \\
\hline $13,015.700 \ldots \ldots \ldots \ldots \ldots \ldots \ldots \ldots \ldots \ldots \ldots \ldots \ldots$ & -26.0176 & 5.3 \\
\hline 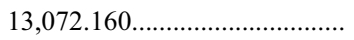 & -29.7505 & 3.9 \\
\hline $13,074.144 \ldots \ldots \ldots \ldots \ldots \ldots \ldots \ldots \ldots$ & -34.2496 & 3.6 \\
\hline $13,077.133 \ldots \ldots \ldots \ldots \ldots \ldots \ldots \ldots$ & -46.1021 & 3.8 \\
\hline
\end{tabular}

$\mathrm{rms}=8.6 \mathrm{~m} \mathrm{~s}^{-1}, \sim 25 \%$ higher than expected from the errors and jitter alone $\left(4 \mathrm{~m} \mathrm{~s}^{-1}\right.$ each). The Keplerian model is adequate, and far superior to the hypothesis of no planet at all. From this simple model, the orbital parameters for the planet are $P=$ $456 \pm 7$ days, $e=0.13$ poorly determined, and $K=33.0 \mathrm{~m}$ $\mathrm{s}^{-1}$, implying a minimum mass $M \sin i=1.30 M_{\text {Jup }}$ orbiting with semimajor axis $a=1.19$ AU.

However, it is also apparent to the eye that the velocities exhibit an upward trend. We thank the anonymous referee for emphasizing this apparent trend. We carried out a Keplerian fit that includes a superposed linear trend, indeed yielding a better fit but nearly identical orbital parameters of $P=456.5$ days, $e=0.15$, $K=32.0 \mathrm{~m} \mathrm{~s}^{-1}, a=1.19 \mathrm{AU}$, and $M \sin i=1.26 M_{\mathrm{Jup}}$, and an upward slope of $3.51 \pm 1.6 \mathrm{~m} \mathrm{~s}^{-1} \mathrm{yr}^{-1}$. The slope is only significant at the $2 \sigma$ level. This model yields residuals with rms of $7.5 \mathrm{~m} \mathrm{~s}^{-1}$ and $\sqrt{\chi_{\nu}^{2}}=1.21$, both smaller than obtained from the model with no trend.

\section{5. $H D 45350$}

HD $45350(=$ HIP 30860) is spectral type G5 V, with $V=7.89, B-V=0.74$, parallax of 20.43 mas (ESA 1997), and absolute visual magnitude $M_{V}=4.44$, placing it $\sim 0.8 \mathrm{mag}$ above the main sequence. It is chromospherically quiet, with $S=0.14$ and $\log R_{\mathrm{HK}}^{\prime}=-5.10$ (Wright et al. 2004). This star was suggested for inclusion in our sample by Laughlin (2000),

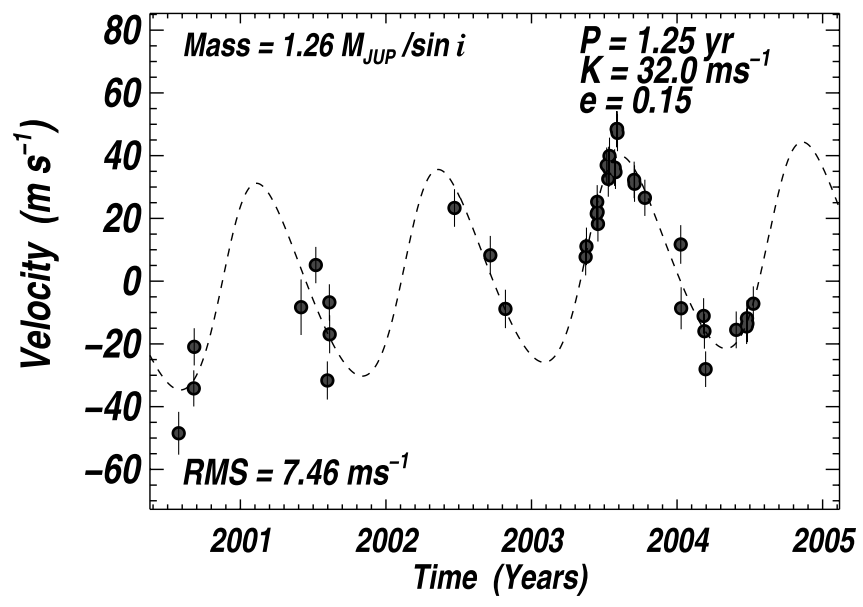

FIG. 5.-Measured velocity vs. time for HD 188015 (dots) with the associated best-fit Keplerian with period $P=456$ days and $M \sin i=1.26 M_{\text {Jup }}$. An upward linear trend is included in the model of $3.51 \mathrm{~m} \mathrm{~s}^{-1} \mathrm{yr}^{-1}$.

who finds it to be metal-rich, $[\mathrm{Fe} / \mathrm{H}]=+0.29$, based on calibration of narrowband photometry. The metallicity was also found to be higher than solar by Valenti \& Fischer (2005), who find $[\mathrm{Fe} / \mathrm{H}]=+0.29$. The implied rotation period is 39 days. Its placement above the main sequence and low chromospheric activity are consistent with an old, quiescent star of age 6-10 Gyr and metal-rich.

We estimated the stellar mass from the Girardi et al. (2002) models, as described in $\S 2$, giving $M=1.02 M_{\odot}$. In comparison, Allende Prieto \& Lambert (1999) find $M=0.98 M_{\odot}$. As our estimate takes account of the high metallicity of the star, we adopt $M=1.02 \pm 0.1 M_{\odot}$. From our spectra of $\mathrm{Ca}$ II $\mathrm{H}$ and $\mathrm{K}$, the expected jitter is $3.86 \mathrm{~m} \mathrm{~s}^{-1}$. Stellar parameters are listed in Table 1.

Table 6 gives the time, the measured radial velocity, and the internal error from each spectrum The expected jitter is $3.86 \mathrm{~m}$ $\mathrm{s}^{-1}$. Figure 6 shows the measured relative velocities for HD 45350 and the associated best-fit Keplerian model. The error bars shown in Figure 6 include both the internal errors and jitter.

The velocities for HD 45350 in Figure 6 reveal it to be varying with peak-to-peak variations of $42 \mathrm{~m} \mathrm{~s}^{-1}$. The error bars shown are the quadrature sum of internal errors and expected jitter, totalling $\sim 6 \mathrm{~m} \mathrm{~s}^{-1}$, implying that the velocity variations represent real motion of the star. The velocities may be fitted successfully with a Keplerian model, but large gaps in orbital phase exist especially near periastron, warranting further observations. The best-fit Keplerian model, including a linear slope, has a $\sqrt{\chi_{\nu}^{2}}=0.87$ and residuals with $\mathrm{rms}=4.55 \mathrm{~m} \mathrm{~s}^{-1}$, indicating that the model is adequate, given the errors and expected jitter. The best-fit slope is only $-0.88 \mathrm{~m} \mathrm{~s}^{-1} \mathrm{yr}^{-1}$, which is only marginally significant, corresponding to a decline of only $4 \mathrm{~m} \mathrm{~s}^{-1}$ during the 4 years of observations. The best-fit orbital parameters for the planet are $P=891 \pm 38$ days, $e=0.78 \pm 0.09$, and $K=33 \mathrm{~m} \mathrm{~s}^{-1}$, implying a minimum mass $M \sin i=0.98 M_{\text {Jup }}$ orbiting with a semimajor axis $a=1.77 \mathrm{AU}$. We note that the eccentricity has a formal uncertainty of 0.09 that comes directly from our Monte Carlo simulations in which we add noise and recompute the best-fit Keplerian orbit for 100 trials. However, the periastron has not been sampled, leaving some concern that the uncertainty may be greater than 0.09 .

\subsection{HD 99492}

HD 99492 (=HIP 55848) is spectral type K2 V, with $V=$ $7.57, B-V=1.01$, parallax of 55.59 mas (ESA 1997), and 
TABLE 6

Radial Velocities for HD 45350

\begin{tabular}{|c|c|c|}
\hline $\mathrm{JD}-2,440,000$ & $\begin{array}{l}\text { Radial Velocity } \\
\qquad\left(\mathrm{m} \mathrm{s}^{-1}\right)\end{array}$ & $\begin{array}{c}\text { Uncertainty } \\
\left(\mathrm{m} \mathrm{s}^{-1}\right)\end{array}$ \\
\hline $11,543.998 \ldots$. & -14.0259 & 3.1 \\
\hline 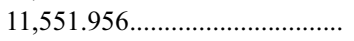 & -13.0124 & 3.5 \\
\hline 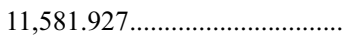 & -10.5125 & 3.5 \\
\hline 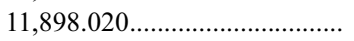 & 26.3054 & 3.2 \\
\hline 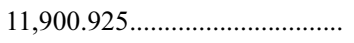 & 24.5338 & 3.2 \\
\hline 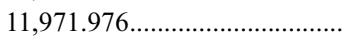 & 7.74938 & 3.7 \\
\hline $11,972.955 \ldots \ldots \ldots \ldots \ldots \ldots . .$. & 13.7434 & 3.7 \\
\hline $11,973.759 \ldots \ldots \ldots \ldots \ldots \ldots \ldots \ldots \ldots$ & 9.54692 & 2.9 \\
\hline $11,974.807 \ldots \ldots \ldots \ldots \ldots \ldots \ldots \ldots \ldots$ & 8.30685 & 3.0 \\
\hline $12,236.044 \ldots \ldots \ldots \ldots \ldots \ldots \ldots \ldots$ & -5.10245 & 3.3 \\
\hline 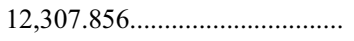 & -1.02283 & 3.6 \\
\hline 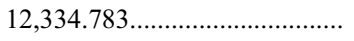 & 0.118599 & 3.6 \\
\hline $12,538.051 \ldots \ldots \ldots \ldots \ldots \ldots \ldots \ldots . .$. & -8.43863 & 3.4 \\
\hline $12,573.062 \ldots$ & -9.02834 & 3.5 \\
\hline 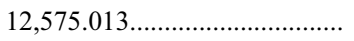 & -3.47303 & 3.7 \\
\hline 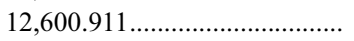 & -12.8009 & 3.9 \\
\hline 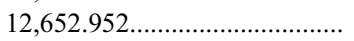 & -16.1870 & 3.7 \\
\hline $12,711.750 \ldots \ldots \ldots \ldots \ldots \ldots \ldots \ldots$. & 4.44637 & 3.6 \\
\hline $12,899.145 \ldots \ldots \ldots \ldots \ldots \ldots \ldots \ldots \ldots . .$. & 15.6017 & 3.3 \\
\hline $12,925.069 \ldots \ldots \ldots \ldots \ldots \ldots \ldots \ldots \ldots$ & 13.7508 & 3.1 \\
\hline $12,987.990 \ldots \ldots \ldots$ & 0.382954 & 2.7 \\
\hline 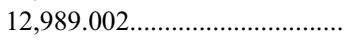 & 0.769168 & 3.7 \\
\hline 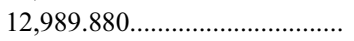 & -0.227416 & 3.3 \\
\hline 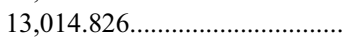 & -2.49941 & 3.3 \\
\hline $13,015.839 \ldots \ldots \ldots \ldots \ldots \ldots \ldots \ldots \ldots$ & -3.69665 & 3.3 \\
\hline 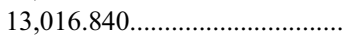 & -4.22298 & 3.3 \\
\hline 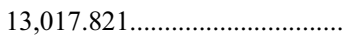 & -1.63862 & 3.4 \\
\hline 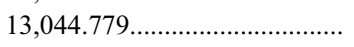 & 1.01771 & 3.3 \\
\hline 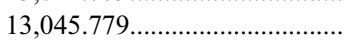 & 5.77902 & 3.2 \\
\hline $13,071.828 \ldots \ldots \ldots \ldots \ldots \ldots$ & 4.77209 & 3.2 \\
\hline
\end{tabular}

absolute visual magnitude $M_{V}=6.30$, placing it $0.3 \mathrm{mag}$ above the main sequence. It is chromospherically average for a $\mathrm{K} 2$ dwarf in the field of the solar neighborhood, with $S=0.27$ and $\log R_{\mathrm{HK}}^{\prime}=-4.93$ (Wright et al. 2004). The implied rotation period is 45 days, but remains uncertain by $\sim 30 \%$ because this star is cooler than the calibrated domain. Valenti \& Fischer (2005) find $[\mathrm{Fe} / \mathrm{H}]=+0.36$, making this yet another planetbearing star with high metallicity. Its placement near the main sequence and its modest chromospheric activity are consistent with a middle-aged star of age 2-6 Gyr.

We estimated the stellar mass from the Girardi et al. (2002) models, as described in $\S 2$, giving $M=0.775 \pm 0.08 M_{\odot}$. Allende Prieto \& Lambert (1999) do not offer a mass estimate for this star. From our spectra of $\mathrm{Ca}$ II $\mathrm{H}$ and $\mathrm{K}$, the expected jitter is $2.50 \mathrm{~m} \mathrm{~s}^{-1}$, low because dwarfs later than $\mathrm{K} 2$ exhibit diminished jitter in general. The stellar parameters are listed in Table 1.

Table 7 gives the time of each observation, the measured velocity, and the internal error. The expected jitter is $2.50 \mathrm{~m} \mathrm{~s}^{-1}$. Figure 7 shows the relative velocities plotted versus orbital phase for HD 99492 and the associated best-fit Keplerian model. The error bars shown in Figure 7 include both the internal errors and predicted jitter.

A periodogram of the velocities for HD 99492 shows a strong peak at a period of 17.0 days, with a false alarm probability well under $1.0 \%$. We adopt this threshold of $1 \%$ false alarm probability (FAP) a priori in the periodogram as a flag to warrant careful and intense follow-up velocity observations, but not of a secure planet detection. In $\S 5$ we describe both an $F$-test and a Monte Carlo test of scrambled velocities that reveal the FAP of

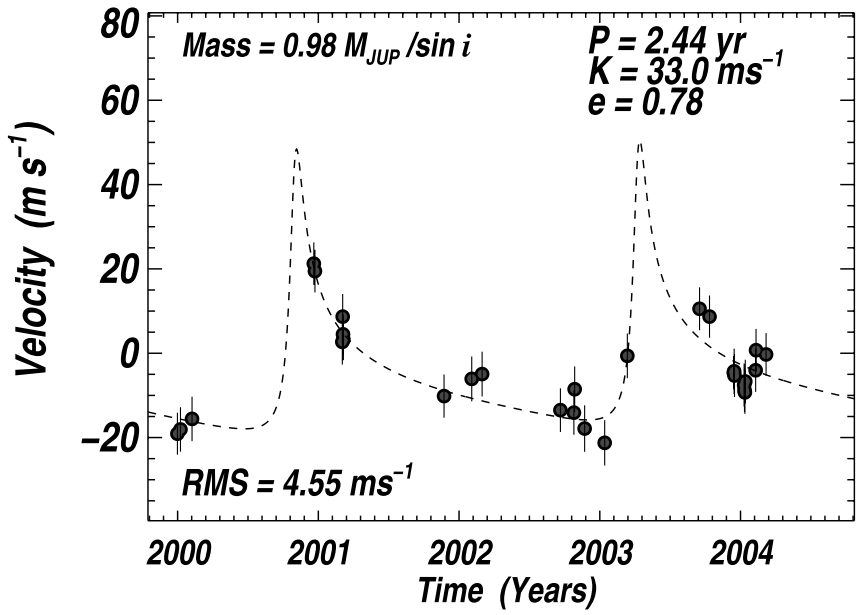

FIG. 6.-Measured velocity vs. time for HD 45350 (dots) with the associated best-fit Keplerian with period $P=891$ days and $M \sin i=0.98 M_{\text {Jup }}$.

the Keplerian orbit to be less than $1 \%$, in agreement with the periodogram result, rendering this a compelling orbital fit.

The velocities shown as a function of orbital phase in Figure 7 reveal clear phase coherence during the 7 years of observations, from 1997 through 2004. The semiamplitude of $10.5 \mathrm{~m} \mathrm{~s}^{-1}$ is

TABLE 7

Radial Velocities for HD 99492

\begin{tabular}{|c|c|c|}
\hline $\mathrm{JD}-2,440,000$ & $\begin{array}{l}\text { Radial Velocity } \\
\qquad\left(\mathrm{m} \mathrm{s}^{-1}\right)\end{array}$ & $\begin{array}{c}\text { Uncertainty } \\
\left(\mathrm{m} \mathrm{s}^{-1}\right)\end{array}$ \\
\hline 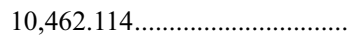 & -5.30884 & 2.2 \\
\hline $10,546.988 \ldots \ldots \ldots \ldots \ldots \ldots \ldots \ldots$ & 1.70639 & 3.2 \\
\hline $10,837.933 \ldots \ldots \ldots \ldots \ldots \ldots \ldots \ldots$ & -10.3165 & 2.8 \\
\hline $10,862.899 \ldots \ldots \ldots \ldots \ldots \ldots \ldots \ldots \ldots$ & -11.8881 & 2.9 \\
\hline 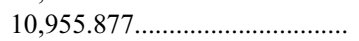 & -6.08888 & 3.0 \\
\hline 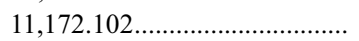 & 0.00000 & 2.4 \\
\hline 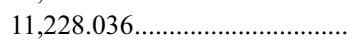 & -10.9208 & 2.9 \\
\hline 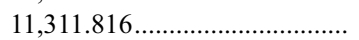 & 7.45052 & 3.3 \\
\hline 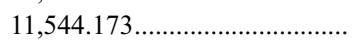 & -9.21701 & 2.8 \\
\hline 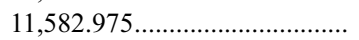 & 0.668655 & 2.6 \\
\hline 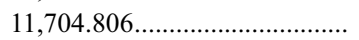 & 4.55197 & 3.8 \\
\hline 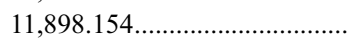 & -13.6692 & 2.9 \\
\hline 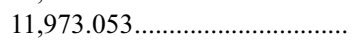 & 2.68497 & 2.9 \\
\hline $12,095.752 \ldots \ldots \ldots \ldots \ldots \ldots \ldots \ldots \ldots \ldots$ & 7.68890 & 3.7 \\
\hline $12,097.754 \ldots \ldots \ldots \ldots \ldots \ldots \ldots \ldots \ldots$ & 3.21675 & 3.7 \\
\hline 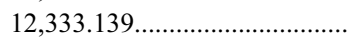 & 6.33837 & 2.9 \\
\hline $12,334.080 \ldots \ldots \ldots \ldots \ldots \ldots \ldots \ldots \ldots \ldots$ & 14.6471 & 3.7 \\
\hline $12,334.968 \ldots \ldots \ldots \ldots \ldots \ldots \ldots \ldots \ldots \ldots$ & 4.63973 & 3.0 \\
\hline $12,364.068 \ldots \ldots \ldots \ldots \ldots \ldots \ldots \ldots \ldots$ & 3.88087 & 3.7 \\
\hline $12,445.768 \ldots \ldots \ldots \ldots \ldots \ldots \ldots \ldots \ldots \ldots$ & -2.76016 & 3.6 \\
\hline 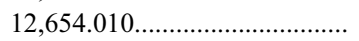 & 7.32495 & 3.0 \\
\hline $12,681.123 \ldots \ldots \ldots \ldots \ldots \ldots \ldots \ldots \ldots \ldots$ & -12.1425 & 3.1 \\
\hline 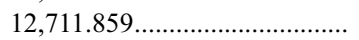 & -0.502421 & 2.9 \\
\hline $12,804.766 \ldots \ldots \ldots \ldots \ldots \ldots \ldots \ldots \ldots$ & -2.67384 & 4.0 \\
\hline $12,805.876 \ldots \ldots \ldots \ldots \ldots \ldots \ldots \ldots \ldots$ & 9.40203 & 4.1 \\
\hline $12,806.764 \ldots \ldots \ldots \ldots \ldots \ldots \ldots \ldots \ldots$ & 2.64147 & 3.5 \\
\hline 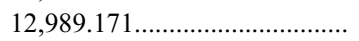 & -10.8823 & 2.7 \\
\hline $13,015.119 \ldots \ldots \ldots \ldots \ldots \ldots \ldots \ldots \ldots \ldots$ & 11.6840 & 3.0 \\
\hline 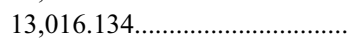 & 7.43558 & 2.8 \\
\hline 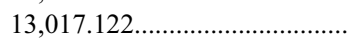 & 6.23335 & 2.7 \\
\hline $13,044.128 \ldots \ldots \ldots \ldots \ldots \ldots \ldots \ldots \ldots$ & -6.25624 & 2.9 \\
\hline 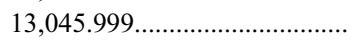 & -0.223535 & 2.8 \\
\hline $13,071.871 \ldots \ldots \ldots \ldots \ldots \ldots \ldots$ & -1.83468 & 2.8 \\
\hline $13,073.941 \ldots \ldots \ldots \ldots \ldots \ldots \ldots \ldots \ldots \ldots$ & -16.2984 & 2.8 \\
\hline $13,076.984 \ldots \ldots \ldots \ldots \ldots \ldots \ldots \ldots \ldots$ & -6.92136 & 2.6 \\
\hline
\end{tabular}




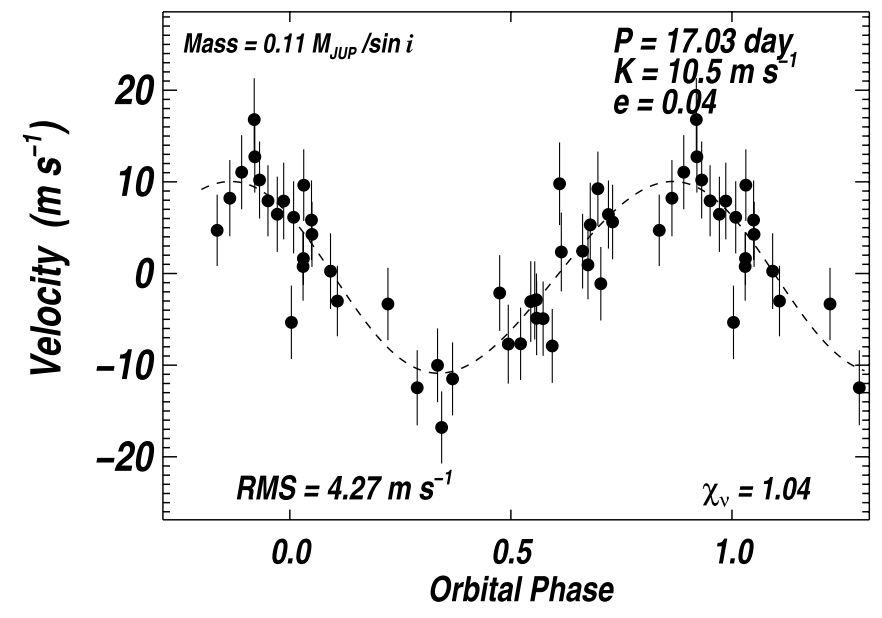

FIG. 7.-Measured velocity vs. orbital phase for HD 99492 (dots) with the associated best-fit Keplerian with period $P=17.038$ days, $K=10.5 \mathrm{~m} \mathrm{~s}^{-1}$, and $M \sin i=0.11 M_{\mathrm{Jup}}$. A linear slope was included in the fit, yielding $1.25 \mathrm{~m}$ $\mathrm{s}^{-1} \mathrm{yr}^{-1}$.

above the internal errors of $2.9 \mathrm{~m} \mathrm{~s}^{-1}$ (median) and the jitter of $2.5 \mathrm{~m} \mathrm{~s}^{-1}$. Thus, the reality of this planet demanded the full 7 years of observation before the FAP from three different tests were all well below $1 \%$. We fit a Keplerian model with a linear slope, yielding $\sqrt{\chi_{\nu}^{2}}=1.10$ and residuals with $\mathrm{rms}=4.3 \mathrm{~m} \mathrm{~s}^{-1}$, both consistent with an adequate model. The Keplerian model is far superior to the hypothesis of no planet at all, as described in $\S 5$ from the $F$-test and Monte Carlo tests. The resulting best-fit orbital parameters for the planet are $P=17.038 \pm 0.011$ days, $e=$ $0.05 \pm 0.12$, poorly determined, $K=10.5 \mathrm{~m} \mathrm{~s}^{-1}$, implying a minimum mass $M \sin i=0.11 M_{\text {Jup }}$ orbiting with semimajor axis, $a=0.12$ AU. The best-fit linear slope is $1.25 \mathrm{~m} \mathrm{~s}^{-1} \mathrm{yr}^{-1}$, which remains marginally significant.

Its companion star, HD 99491, exhibits a velocity rms of $5.99 \mathrm{~m} \mathrm{~s}^{-1}$ in $27 \mathrm{Keck} / \mathrm{HIRES}$ spectra obtained from 1997.1 to 2004.0. This rms is consistent with its internal errors of $2.9 \mathrm{~m}$ $\mathrm{s}^{-1}$ (median) and predicted jitter of $5.89 \mathrm{~m} \mathrm{~s}^{-1}$. No real velocity variation is apparent, and it shows neither periodic power nor a compelling Keplerian fit.

\section{FALSE ALARM PROBABILITY}

We wish to assess the probability that the best Keplerian model fitted to a set of velocities, and the associated $\chi_{\nu}^{2}$ statistic, could have arisen simply as a result of noise fluctuations. Such a false alarm diagnostic is especially desirable for Keplerian curves having amplitudes comparable to the noise in the velocity measurements. Arbitrarily small Keplerian amplitudes (and hence arbitrarily small planet masses) may be detected by obtaining enough velocity measurements having known uncertainties. We need to quantify the probability that velocity fluctuations due to noise can masquerade as Keplerian variations.

For periodic phenomena, a special danger lies in fitting a time series of data with models having short orbital periods. The danger lies both in identifying the shortest detectable orbital period and in the large number of distinct (short) periods that can be tailored to the data. The shortest orbital period that can be detected in a given set of velocity measurements is not mathematically sharp, as the Nyquist frequency is poorly defined for unevenly spaced velocity measurements. One may define a period corresponding to the nominal Nyquist frequency, $P_{\mathrm{N}}=$ $2 T / N_{\text {obs }}$, where $T$ is the duration of observations and $N_{\text {obs }}$ is the number of observations. However, observations that are sepa- rated by fractional amounts of that nominal Nyquist period provide information about periods much shorter than that nominal Nyquist limit (A. Cumming 2005, in preparation). Moreover, a signal with an amplitude well above the noise level will be readily apparent independent of period, albeit with aliasing.

The independent periods that can be distinguished uniquely in a time series represent separate statistical states in which fluctuations may mimic a true signal. The spacing of distinguishable periods is set by adjacent periods that differ just enough to accumulate a phase difference of order one cycle during the full duration of the time series. The density of distinguishable periods increases with decreasing period, with a spacing that depends quadratically on period, $\Delta P \propto P^{2}$. For this reason, power spectra and periodograms are commonly sampled with equal spacing in frequency rather than period (Scargle 1982; Horne \& Baliunas 1986; Gilliland \& Baliunas 1987).

For our case of fitting orbits to velocity measurements, the high density of distinguishable Keplerian states at short periods implies a higher probability at such periods that noise fluctuations will yield a spurious Keplerian fit despite yielding $\chi_{\nu}^{2} \sim$ 1. Indeed, we commonly find good Keplerian fits with periods under $\sim 10$ days, later revealed to be spurious with more measurements.

We require a test that allows us to quantitatively assess the hypothesis that noise alone could have caused an apparently "good" Keplerian fit by mere fluctuations of noise. We have carried out two such tests, one using the $F$-statistic and the other using Monte Carlo simulations. Excellent treatment of the detectability of Keplerian signals has been provided by Loredo \& Chernoff (2003), Cumming et al. (2003), Ford (2005), and A. Cumming (2005, in preparation).

\subsection{F-Test}

A "frequentist" approach to false alarm testing involves assessing the probability that a value of $\chi_{\nu}^{2}$ would occur that is lower than that from the best-fitting model simply because of fluctuations in noise in the data, rather than caused by an actual signal. The $F$-test provides a good diagnostic of FAP under the assumption that the errors of the measurements have a Gaussian distribution (Bevington \& Robinson 1992). For the case of Keplerian fitting, the $F$-test determines the probability that a set of velocities would yield a $\chi^{2}$ statistic from a Keplerian fit as low or lower than that from the "no planet" (noise-only) model. A formal description of the $F$-test for the detectability of extrasolar planets was derived by Cumming et al. (2003; A. Cumming 2005, in preparation). We follow that approach here.

Stars often exhibit constant acceleration (radial velocity trends) caused by long-period companions. We thus first fit a straight line to the velocities and compute the $\chi^{2}$ statistic for that "trend but no planet" model. We then fit a Keplerian model to the residual velocities, yielding its $\chi^{2}$. The $F$-test determines the probability that one model constructed with additional free parameters is to be preferred over another model, as a function of the decrease in their respective $\chi_{\nu}^{2}$ (Bevington \& Robinson 1992; A. Cumming 2005, in preparation).

A complication arises because of the large number of plausible and distinguishable orbital periods, at each of which noise fluctuations could conspire to yield a good Keplerian fit. An apparently low value of $\chi^{2}$ could arise by chance fluctuations from the wide selection of such available orbital periods at which fluctuations can occur. This wide selection is analogous to the proper assessment of FAP in periodogram analyses (Horne \& Baliunas 1986; Gilliland \& Baliunas 1987) in which the number of independent frequencies must be counted. Any 
of the $N_{i}$ independent frequencies could harbor chance power in the periodogram coming from noise only. Similarly in the $F$-test applied to Keplerian models, one must include the $N_{i}$ distinguishable orbital periods.

We define $\operatorname{FAP}_{P}$ to be the probability that pure noise data would yield a $\chi^{2}$ as low or lower than that actually found, $\chi_{\text {best }}^{2}$, at one specific period $P$ from the best fit. $\mathrm{FAP}_{P}$ depends on the difference between $\chi_{\text {best }}^{2}$ and $\chi^{2}$ from a "trend but no planet" model (A. Cumming 2005, in preparation). The probability that, at one period, chance fluctuations would not yield a $\chi^{2}$ as low or lower than $\chi_{\text {best }}^{2}$ is $1-\mathrm{FAP}_{P}$, and hence the probability that none of the independent frequencies have fluctuations giving such a low FAP is $\mathrm{FAP}_{\text {none }}=\left(1-\mathrm{FAP}_{P}\right)^{N_{i}}$. The converse, namely, that at least one independent frequency does have a fluctuation that gives a $\chi^{2}$ as low or lower than $\chi_{\text {best }}^{2}$ is

$$
\mathrm{FAP}_{\text {any } P}=1-\left(1-\mathrm{FAP}_{P}\right)^{N_{i}}
$$

The nonuniform spacing of observations gives access to periods as short as 1 day, leading A. Cumming (2005, in preparation) to find that $N_{i} \approx 2 T$, where $T$ is the duration of observations in days. This value of $N_{i}$ comes from the spacing of periods that are distinguishable by the accumulated phase difference between adjacent periods. We note that this of $N_{i}$ is typically much greater than that traditionally used for periodogram analyses, the difference stemming from the sub-Nyquist periods being sampled here. These additional periods add to the number of possible false alarms.

A benchmark case is the regime of very low false alarm probability, $\operatorname{FAP}_{P} \ll 1$, for which the binomial expansion yields

$$
\mathrm{FAP}_{\text {any } P}=N_{i} \times \mathrm{FAP}_{P}
$$

This agrees with one's intuition that if the FAP for one period is much less than unity, then the chance of a false alarm occurring at any of the $N_{i}$ periods is the sum of the probabilities over all independent periods. For example, if there is a $0.1 \%$ FAP at one period, then for 10 independent periods there are 10 opportunities for a spurious signal, leading to a total FAP of $1 \%$.

We set a threshold a priori of FAP $<0.01$ for suggesting that a Keplerian signal exists in the velocity measurements. We have applied this $F$-test to all five planets reported here, and all five have FAP $<0.01$. Four stars gave FAP $<10^{-5}$. However, two were questionable, namely, HD 45350b and HD 99492b, which gave an FAP of 0.00004 and 0.0003 , respectively. Thus, all five stars exhibit velocities for which noise is unlikely to be the cause of the apparent Keplerian velocity variations.

With 975 stars on the planet search survey, even an a priori threshold FAP of $1 \%$ does not provide compelling evidence that the orbits are real. Fluctuations may produce spurious signals at an FAP threshold of $1 \%$ out of such a large stellar sample. To date, however, no false claims have emerged from the 75 planets we have announced from our three planet surveys at Keck, Lick, and the Anglo-Australian Telescope (AAT). We suspect that adhering to a strict $1 \%$ FAP will become more important in the future. Most of the remaining undetected planets orbiting nearby FGK stars $(d<30 \mathrm{pc})$ either have masses less than $1 M_{\text {Jup }}$ or reside in orbits beyond $3 \mathrm{AU}$, thus straining our ability to provide secure detections. This FAP threshold of $1 \%$ may be a useful benchmark for credibility.

After some velocity measurements are obtained, one Keplerian orbit may yield a low FAP, rendering it a likely model. This candidate model collapses the value of $N_{i}$ to 1 , as all other periods are no longer under scrutiny. This collapse marks a "prediction" to be confirmed or rejected by further data. After such data are collected, the final FAP of the entire data set is the product of two FAPs, namely, that prior to prediction and that after prediction. The latter is computed in the same way, but using only the later data points and using $N_{i}=1$, giving those new data more power in the test.

Three weaknesses of the $F$-test are: its assumption of Gaussian-distributed velocity errors, its inability to account for the actual uneven temporal spacing of the observations, and its dependence on $N_{i}$ that is set only approximately. The FAP from the $F$-test can serve to flag stars that warrant a more robust test, albeit CPU-intensive, that suffers from fewer weaknesses.

\subsection{FAP Assessment: The Scrambled Velocities Approach}

A more robust, albeit brute force, frequentist approach to determine the FAP of a prospective orbital fit involves a Monte Carlo scrambling of the velocities. We adopt the null hypothesis that the velocity measurements are merely incoherent noise. The hypothesis is tested by scrambling the order of the velocities and comparing the original set to the scrambled velocities for evidence of Keplerian coherence.

This FAP assessment makes no demands on the distribution of the noise, unlike the $F$-test, which is accurate only for Gaussian errors. Indeed, velocity noise is unlikely to follow a Gaussian distribution because it stems both from Poisson photon statistics in the spectrum and from flaws in the Doppler analysis such as imperfect treatment of the spectrometer PSF and poor template deconvolution. Moreover, velocity jitter from the photosphere contributes a non-Gaussian noise component.

We proceed by first removing any linear trend in the original velocity measurements as a function of time, using only the residuals to a linear fit. This permits coherence to be detected despite the presence of any superposed long-term trend in the velocities. The order of the velocity residuals is scrambled, using a pseudo-random number generator, keeping the times of observation the same. This generates a set of velocities with identical temporal sampling but without the original temporal coherence, if any were present. We generate 1000 such sets of velocities, and for each we carry out a blind and thorough search for the best Keplerian model, yielding a best-fit value for $\chi_{\nu}^{2}$.

The resulting values of $\chi_{\nu}^{2}$ from the scrambled velocities may be compared to the $\chi_{\nu}^{2}$ that stemmed from the original, unscrambled velocities. If a Keplerian signal is present in the original velocities, the $\chi_{\nu}^{2}$ from the Keplerian fit will tend to be lower than the $\chi_{\nu}^{2}$ values coming from most of the scrambled velocities. The $\chi_{\nu}^{2}$ from scrambled velocities is higher because of the destruction of orbital phase coherence in the original velocities, if any. The FAP is the fraction of trials of scrambled velocities that nonetheless yield lower $\chi_{\nu}^{2}$ than from the original set. This FAP specifies the probability that mere noise could have yielded a superior Keplerian fit by chance.

No assumptions are made in this "scrambled velocity" FAP test about the expected distribution or the magnitude of the noise. Doppler measurements for a particular star could exhibit a larger than expected noise because of poorly anticipated Doppler errors, photospheric jitter, or rare noise sources that are uncorrelated in time. In such cases the value of $\chi_{\nu}^{2}$ will still tend to be lower than the original velocities if they are temporally coherent compared with those from the scrambled velocities. Thus, the scrambled velocity method is resistant to poor estimates of internal velocity errors and jitter.

The scrambled velocity method is sensitive to Keplerian velocity amplitudes, $K$, that are comparable to or less than the velocity errors and jitter. As more velocities are measured that 


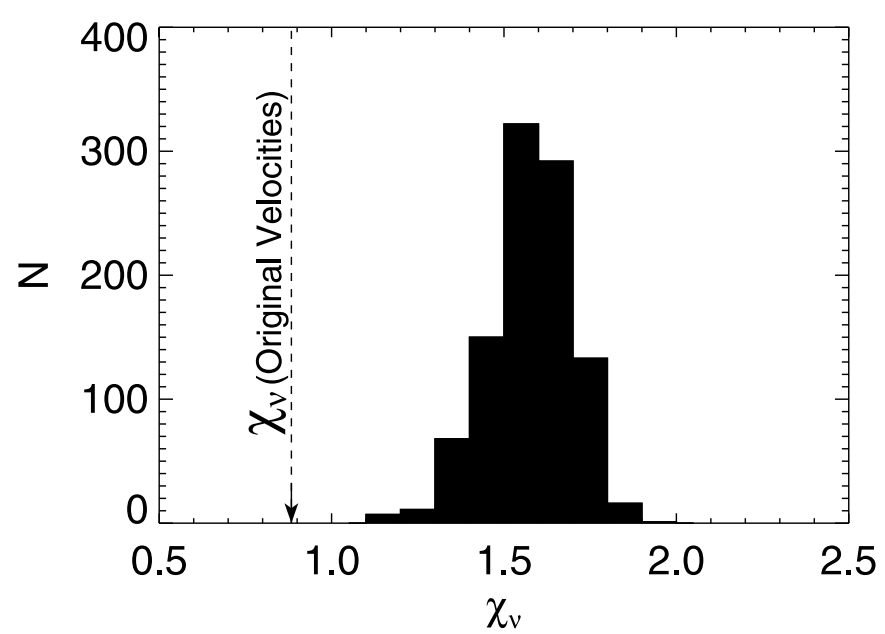

FIG. 8.-Assessment of FAP of the Keplerian model for HD 45350. The histogram shows the values of $\sqrt{\chi_{\nu}^{2}}$ from Keplerian fits to 1000 sets of scrambled velocities. The original, unscrambled velocities yield $\sqrt{\chi_{\nu}^{2}}$ (vertical dashed line), clearly lower than from the scrambled velocities. The lower value of $\chi_{\nu}^{2}$ from the original velocities indicates the presence of temporal coherence consistent with Keplerian motion. None of the 1000 sets of scrambled velocities yielded $\chi_{\nu}^{2}$ as low as from the original velocity set, indicating that FAP $<0.001$. Fluctuations in velocity noise are unlikely to explain the good Keplerian fit to the observed velocities.

contain a real signal, the resulting $\chi_{\nu}^{2}$ will emerge as lower than the $\chi_{\nu}^{2}$ values from the scrambled velocities, with the FAP diminishing accordingly. The sensitivity to the phase coherence in the Keplerian velocities delivers a decreasing FAP as more measurements are made. This FAP test is differential, as it simply compares the fits from the original velocities to those scrambled, both treated identically.

There are similarities between the scrambled velocity test and the $F$-test described in $\S 5.1$. The large number of independent periods, $N_{i}$, that had to be anticipated awkwardly with the $F$-test is accounted for naturally in the Monte Carlo method. The full range of possible orbital periods is always surveyed in each fit of the scrambled velocities. With both the scrambled velocity test and $F$-test, a predicted Keplerian orbit can emerge at some stage during the experiment. As with the $F$-test, this prediction offers the opportunity to compute a separate, postprediction FAP directly from the value of $\chi_{\nu}^{2}$, with the period collapsed to only one possibility. Finally, there is no safeguard in either FAP test against intrinsic periodicities, such as from jitter or spot cycles, that can mimic a Keplerian velocity coherence.

We applied this Monte Carlo approach to assess the FAP for the two stars for which the planet interpretation is questionable, namely, HD 45350 and 99492 . We scrambled the velocities, keeping the times of observation the same. For each of 1000 realizations, we searched for the best-fit Keplerian model and recorded its associated value of $\chi_{\nu}^{2}$. The resulting histograms of $\chi_{\nu}^{2}$ are shown in Figures 8 and 9 , respectively. These show the distribution of $\chi_{\nu}^{2}$ expected if the measured velocities were simply uncorrelated noise. For both stars, the entire distribution of $\chi_{\nu}^{2}$ from scrambled velocities is higher than that for the original, unscrambled velocities. For HD 45350, none of the 1000 trials of scrambled velocities yielded $\chi_{\nu}^{2}$ as low as that found in the fit to the actual velocities. For HD 99492, only one of the 1000 trials yielded $\chi_{\nu}^{2}$ as low as that found in the fit to the actual velocities. This shows that the FAP of the Keplerian fit is well under $1 \%$ for both stars, and it is probably no more than $\sim 0.1 \%$ for both of them. These values of FAP are consistent with those derived from the $F$-test, described in $\S 5.1$.

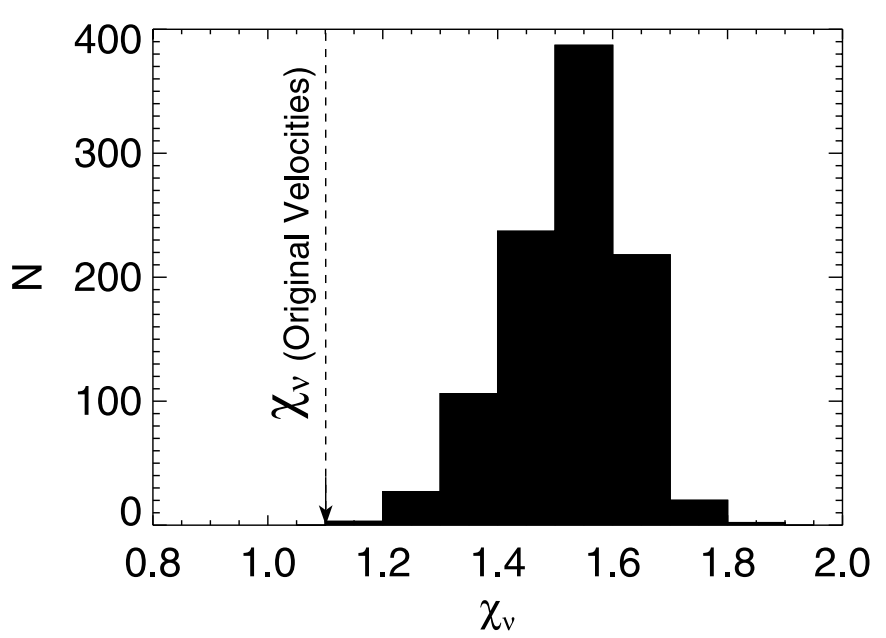

FIG. 9.-Assessment of FAP of the Keplerian model for HD 99492. As with Fig. 10, the histogram and vertical line represent the $\sqrt{\chi_{\nu}^{2}}$ values from scrambled and original velocities, respectively. Only one of the 1000 sets of scrambled velocities yielded $\chi_{\nu}^{2}$ as low as from the original set, giving FAP $\approx$ 0.001 . It is unlikely that chance fluctuations in noise can explain the low $\chi_{\nu}^{2}$ obtained in the Keplerian fit to the actual (unscrambled) velocities.

This scrambled velocity analysis suggests directly that fluctuations in noise are unlikely to account for the low $\chi_{\nu}^{2}$ from the Keplerian fits for HD 45350 and HD 99492. Instead, the velocities apparently display a Keplerian coherence resulting in the low value of $\chi_{\nu}^{2}$. These two planet candidates are unlikely to be due to mere fluctuations in the velocity noise.

\subsection{Spurious Periodicities}

Temporal correlations in the data of any origin may mimic a Keplerian signal. The assessment of FAP from the scrambled velocity FAP test cannot sense a false alarm caused by a periodicity of non-Keplerian origin. Any systematic error or astrophysical effect that recurs on some timescale and that remains coherent during the 5 years of observations can masquerade as the Keplerian signal. Prospective signals that have an amplitude less than $\sim 40 \mathrm{~m} \mathrm{~s}^{-1}$ are so weak that periodicities of nearly any shape, sinusoidal or otherwise, could be adequately fitted with a Keplerian model. Two conceivable sources of such periodicities are changes in the spectrometer that persist for 5 years and periodicities in the structure or atmosphere of the star itself.

One specific concern stems from inhomogeneities such as spots or magnetic plage on the stellar surface whose visibility is modulated by rotation of the star. As shown by Queloz et al. (2001) and Paulson et al. (2004), such photospheric features can result in periodic radial velocity variations capable of mimicking the presence of a planetary companion. Photometric measurements of sufficient quality can detect the associated brightness modulation of planetary candidate host stars, if present, and thus help to establish whether the radial velocity variations are caused by stellar magnetic activity or planetary-reflex motion (e.g., Henry et al. 2000a). Photometric observations are also important to search for transits of the planetary companions (e.g., Henry et al. 2000b). Our photometric results for four of the five host stars in this paper are presented in the next section.

\section{PHOTOMETRY OF THE HOST STARS}

We have obtained photometry of four of the five planetary host stars in this paper between 2002 September and 2004 July with the T12 $0.8 \mathrm{~m}$ automatic photometric telescope (APT) at Fairborn Observatory. The T12 APT is equipped with a 


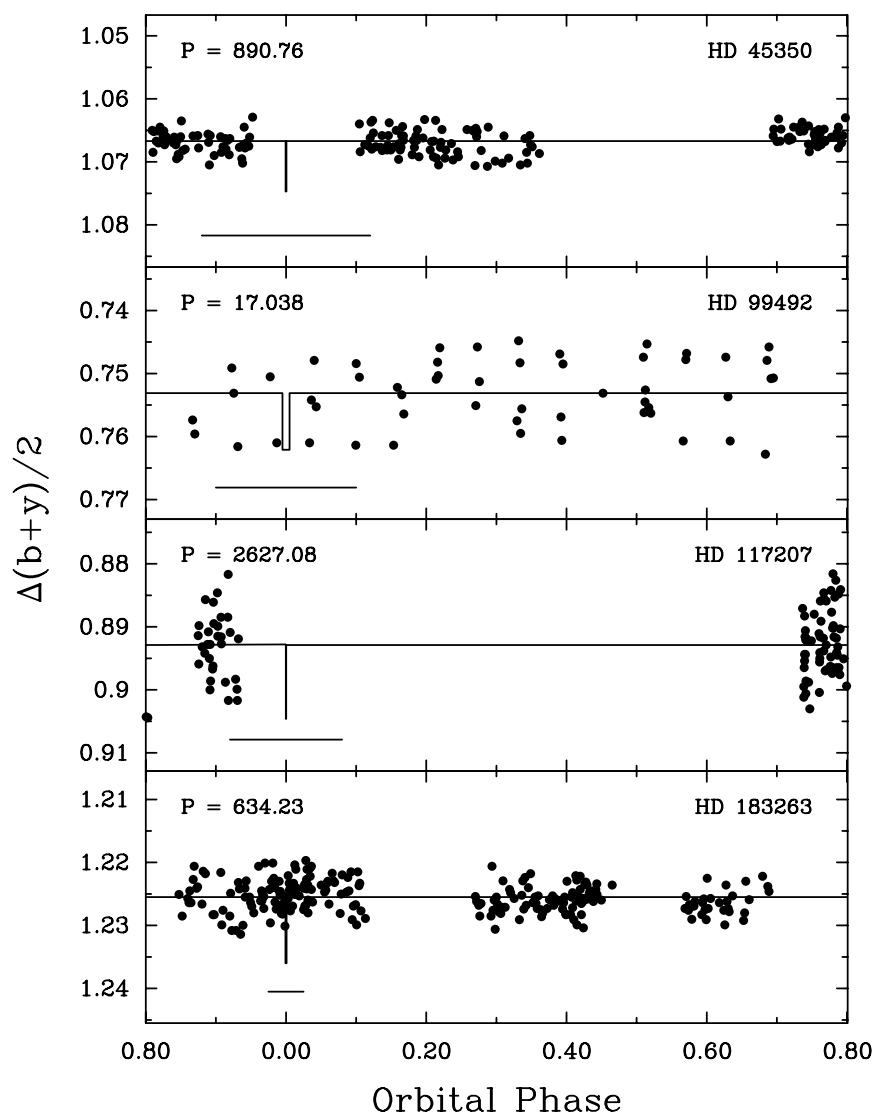

FIG. 10.-Photometric observations of four of the five planetary host stars plotted vs. orbital phase of the planet, with times of inferior conjunction (midtransit) at phase 0.0 . The solid line shows the mean photometric level, with a box function drop at the time of predicted transit. The bar beneath indicates the uncertainty in the time of transit, from the Doppler-based Keplerian orbit. Transits are not observed in any of the four stars, but are not ruled out for the first three stars. The observations around the predicted time of transit for HD 183263 are replotted in Fig. 11 for better visibility. The lack of brightness variability on the radial velocity periods in any of the stars supports planetary-reflex motion as the cause of the velocity variations.

two-channel precision photometer employing two EMI 9124QB bialkali photomultiplier tubes to make simultaneous measurements in the Strömgren $b$ and $y$ passbands. The APT measures the difference in brightness between a program star and a nearby comparison star that has been demonstrated to be constant. The telescope, photometer, observing procedures, and data reduction techniques employed with this APT are very similar to those for the T8 $0.8 \mathrm{~m}$ APT described in Henry (1999). Further details on the development and operation of the automated telescopes can be found in Henry (1995a, 1995b) and Eaton et al. (2003).
Our Strömgren $b$ and $y$ differential magnitudes have been corrected for differential extinction with nightly extinction coefficients and transformed to the Strömgren system with yearly mean transformation coefficients. The external precision of the differential magnitudes is typically around $0.0015 \mathrm{mag}$ for this telescope, as determined from observations of pairs of constant stars. The local comparison stars used for each program star were HD 48412 (for HD 45350), HD 101730 (for HD 99492), HD 117718 (for HD 117207), and HD 183085 (for HD 183263); all four comparison stars are constant to $\sim 0.002$ mag or better, as determined by intercomparison with additional comparison stars.

The combined $(b+y) / 2$ differential magnitudes for each star are plotted in the four panels of Figure 10. The observations are phased with the planetary orbital period and a time of inferior conjunction, computed in each case from the orbital elements in Table 2. The solid curve in each panel approximates the predicted transit light curve assuming a planetary orbital inclination of $90^{\circ}$ (central transits). The out-of-transit light level corresponds to the mean brightness of the observations. The transit durations are calculated from the orbital elements, while the transit depths are derived from the estimated stellar radii, and the planetary radii from the models of Bodenheimer et al. (2003). The horizontal bar below each predicted transit curve represents the approximate $\pm 1 \sigma$ uncertainty in the time of midtransit, based on Monte Carlo simulations and the uncertainties in the orbital elements.

The results from the photometric observations are summarized in Table 8 . The standard deviations in column (4) refer to the spread of the $(b+y) / 2$ measurements around the mean values of the data sets. Two stars, HD 99492 and HD 117207 , have somewhat elevated standard deviations due to a bright, close companion in the first case and the high air mass of the observations in the second. All standard deviations are consistent with the constancy of the host stars; periodogram analyses also failed to reveal significant periodicities in any of the four stars. Column (5) lists the semiamplitudes of the light curves computed from least-squares sine fits of the observations phased to the radial velocity periods. With the exception of HD 117207, whose observations cover too small a portion of the phase curve, these amplitudes are negligible and do not indicate brightness variability on the radial velocity periods. Therefore, the photometric results support planetary-reflex motion as the cause of the radial velocity variations.

Column (6) of Table 8 gives the geometric probability of transits assuming random orbital inclinations, computed from equation (1) of Seagroves et al. (2003). The transit probabilities are all small and, with the exception of HD 99492, less that $1 \%$. As shown by the horizontal bars in Figure 10, the uncertainties in the times of transits are much larger than their calculated

TABLE 8

Photometric Results for the Planetary Host Stars

\begin{tabular}{|c|c|c|c|c|c|c|}
\hline $\begin{array}{l}\text { Star } \\
(1)\end{array}$ & $\begin{array}{c}\text { Date Range } \\
(\text { HJD }-2,450,000) \\
(2)\end{array}$ & $\begin{array}{c}N_{\text {obs }} \\
(3)\end{array}$ & $\begin{array}{c}\sigma \\
(\mathrm{mag}) \\
(4)\end{array}$ & $\begin{array}{l}\text { Semiamplitude } \\
\text { (mag) } \\
\text { (5) }\end{array}$ & $\begin{array}{c}\text { Transit Probability } \\
\qquad(\%) \\
(6)\end{array}$ & $\begin{array}{c}\text { Transits } \\
\text { (7) }\end{array}$ \\
\hline HD $45350 \ldots \ldots \ldots . .$. & $2532-3126$ & 170 & 0.0018 & $0.0005 \pm 0.0002$ & 0.5 & $?$ \\
\hline HD 99492 ........... & $3122-3189$ & 53 & $0.0053^{\mathrm{a}}$ & $0.0015 \pm 0.0010$ & 3.5 & $?$ \\
\hline HD $117207 \ldots \ldots \ldots . .$. & $2645-3158$ & 93 & $0.0053^{\mathrm{b}}$ & $\ldots$ & 0.2 & $?$ \\
\hline HD $183263 \ldots \ldots .$. & $2532-3191$ & 238 & 0.0024 & $0.0006 \pm 0.0002$ & 0.3 & Unlikely \\
\hline
\end{tabular}

a Sigma elevated because nearby companion is 1.1 mag brighter and $28^{\prime \prime}$ away.

b Sigma elevated because of the extreme southern declination of $-36^{\circ}$. 


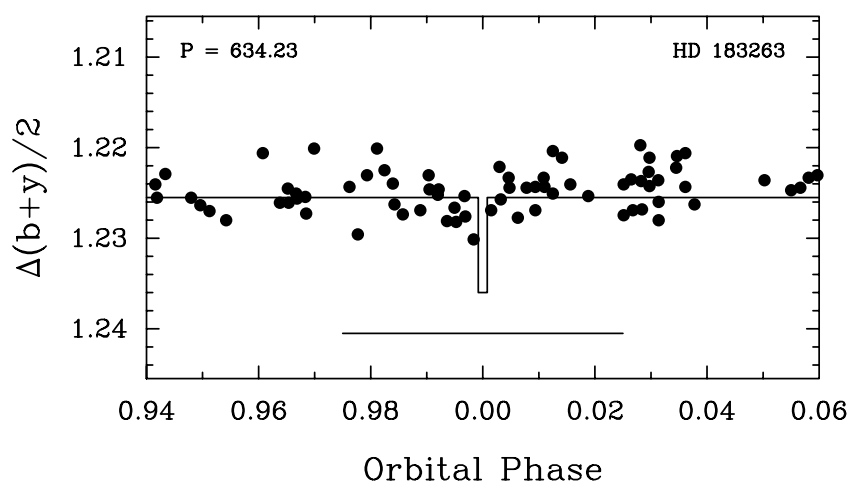

FIG. 11.-Photometric observations of HD 183263 around the predicted time of mid-transit. While transits somewhere within the uncertainty interval cannot be completely ruled out, the existing photometry makes them unlikely.

durations because of the low radial velocity amplitudes and small number of orbital cycles observed so far. We have obtained photometric observations within the uncertainty intervals for all four stars. No transits have been detected, but they are clearly not ruled out for HD 45350, HD 99492, and HD 117207 because of poor phase coverage. The observations around the predicted time of transit for HD 183263 have been replotted with an expanded abscissa in Figure 11. Transits somewhere within the uncertainty interval cannot be completely ruled out, but they are rendered fairly unlikely by the existing photometry.

\section{DISCUSSION}

From the survey of 1330 FGKM main-sequence stars carried out on the Lick, Keck, and AAT telescopes, 77 extrasolar planets had emerged previously. The five secure planets described here brings the total to 82 , bringing more statistical weight to the ensemble. Details of the Doppler measurements and analyses for all 77 previous planet candidates can be found in a series of papers, notably Butler et al. (2002), in their Table 1, and subsequent discoveries (Vogt et al. 2002; Marcy et al. 2002; Butler et al. 2003; Tinney et al. 2003; Jones et al. 2003; Carter et al. 2003; Fischer et al. 2003a, 2003b; McCarthy et al. 2004). A few of these 77 planets were announced first or simultaneously by other teams, notably Mayor \& Santos (2003) and Cochran et al. (1997). Good agreement exists among the measured orbital parameters found by different groups, typically within $10 \%$. Here we focus our attention on the 82 planets found by our group at Lick, Keck, and the AAT because of the uniform Doppler precision of $3 \mathrm{~m} \mathrm{~s}^{-1}$ and the known selection effects of the stellar sample published in Wright et al. (2004) and Nidever et al. (2002).

The extrasolar planets reveal a mass distribution that rises steeply toward lower masses, $d N / d M \propto M^{-1.3}$ (Jorissen et al. 2001; Marcy et al. 2005). The distribution of planets with semimajor axes reveals a rising number toward greater orbital distances when binned in logarithmic intervals of $a$ (Marcy et al. 2005). The orbital eccentricities are distributed nearly uniformly from $e=0$ to 0.7 . These planetary properties agree with those found by Mayor \& Santos (2003).

Four of the five planets presented here have semimajor axes beyond $1 \mathrm{AU}$, continuing a trend for the planets discovered in the past 3 years. Among the nearest FGK main-sequence stars $(d<40 \mathrm{pc})$ the yet undiscovered giant planets typically reside beyond $1 \mathrm{AU}$ because the giant planets within $1 \mathrm{AU}$ have already been found. The distribution of semimajor axes, $d N / d \log a$, increases with increasing semimajor axis (Marcy et al. 2005), a trend that is strengthened by the four of six planets here that reside beyond $1 \mathrm{AU}$. The increasing numbers of planets with orbital distance (in logarithmic bins) suggests that a reservoir of Jupiter-mass planets resides yet farther out, at 3-10 AU, roughly equal in number to the planets discovered inward of $3 \mathrm{AU}$ so far. If so, the occurrence rate of giant planets within $10 \mathrm{AU}$ that are more massive than Saturn appears to be $\sim 15 \%$.

To date, velocity precision of $3 \mathrm{~m} \mathrm{~s}^{-1}$ limits the detected velocity semiamplitudes to greater than $\sim 10 \mathrm{~m} \mathrm{~s}^{-1}$ unless many orbits have transpired. This limit in precision along with the $10 \mathrm{yr}$ lifetime of most surveys establishes the discovery parameter space in planet mass and semimajor axis. As Doppler precision improves and duration of observations increases, more planets are being discovered at larger semimajor axes, with the majority of newly discovered planets residing beyond $1 \mathrm{AU}$. In the past few years, more planets of lower mass are being discovered, with 10 planets having $M \sin i$ below $1 M_{\text {Sat }}$, namely, HD 16141 and HD 46375 (Marcy et al. 2000), HD 168746 (Pepe et al. 2002), HD 76700 (Tinney et al. 2003), HD 49674 (Butler et al. 2003), $55 \mathrm{Cnc}$ c (Marcy et al. 2002), and HD 3651 (Fischer et al. $2003 \mathrm{a}$ ), along with the three planets having $M \sin i$ near $1 M_{\mathrm{Nep}}$ (Butler et al. 2004; McArthur et al. 2004; Santos et al. 2004b). All known sub-Saturn candidates have orbital periods less than 75 days, a limit that probably stems from detectability rather than reality. Here, we add the planet around HD 99492 to the list of sub-Saturn-mass candidates with its minimum mass of $M \sin i=$ $0.11 M_{\text {Jup }}$. These detections of low-mass planets for which detectability is poor reinforces the suggestion that the planet mass function rises even more rapidly toward lower masses than $d N / d M \propto M^{-1}$.

While detectability may explain both the lack of sub-Saturn planet masses orbiting beyond a semimajor axis of $0.35 \mathrm{AU}$ and the paucity of sub-Jupiter masses beyond $0.35 \mathrm{AU}$, inward of that orbital distance sub-Jupiter candidates are found commonly. Inward of $0.35 \mathrm{AU}, 22$ of 35 known extrasolar planets have $M \sin i<1 M_{\text {Jup }}$. Beyond $0.35 \mathrm{AU}$, the known planets typically have $M \sin i>1 M_{\mathrm{Jup}}$, a mass cutoff influenced, no doubt, by detectability. Planets less massive than $1 M_{\text {Jup }}$ orbiting beyond $0.5 \mathrm{AU}$ remain difficult to detect, although not impossible. In particular, planets having mass $0.5 M_{\text {Jup }}$ orbiting at $0.5 \mathrm{AU}$ induce a velocity semiamplitude of only $\sim 20 \sin i \mathrm{~m} \mathrm{~s}^{-1}$, small enough to be missed in present Doppler surveys, depending on the sampling frequency, the jitter, and the duration of observations compared to the period. Thus, the majority of subJupiters are found within $0.5 \mathrm{AU}$, where the induced velocity signal is greater and several orbits have transpired. The difference in the apparent mass distribution between planets close and far from the host star has led some to conclude (Udry et al. 2003) that planet mass distribution depends on semimajor axis, carrying implications for migration theories. However, such conclusions must be viewed with caution as detectability effects operate in the same sense. That the mass distribution for close-in planets appears shifted to lower masses compared to the mass distribution for planets orbiting farther away could result from the detection bias against low-mass planets farther out.

Indeed, among the five planet candidates presented here, only one has $M \sin i<0.5 M_{\text {Jup }}$, namely, HD 99492, which has $M \sin i=0.11 M_{\mathrm{Jup}}, P=17$ days, and $a=0.11 \mathrm{AU}$. The difficulty in detecting this planet $\left(K=10.5 \mathrm{~m} \mathrm{~s}^{-1}\right)$ highlights the aforementioned detection effects that confine the discovery of sub-Jupiter-mass planets mostly to within $0.5 \mathrm{AU}$.

The origin of orbital eccentricities in extrasolar planets remains unclear. Particularly noteworthy are the many extrasolar planets in eccentric orbits that appear to be single, with no other 
Jupiter-mass planets orbiting within $5 \mathrm{AU}$. Two good examples are 70 Vir and HD 222582 (Butler et al. 2002). However, presented here are two planets having $e>0.3$, namely, HD 183263 and HD 45350, both of which reveal evidence of long-term trends in the velocities, indicating the possible presence of a more distant companion. Further observations will be necessary to determine if they indeed have an outer companion or if instead they add to the class of single planets in eccentric orbits. The precise occurrence rate of outer companions that orbit beyond planets that are in eccentric orbits will provide empirical lynchpins for the various models that attempt to explain those orbital eccentricities. The presence of a second giant planet, in the form of Keplerian variation or long-term trends in the velocities, adds weight to models that invoke other giant planets to perturb the observed planet into its eccentric orbit (Marzari \& Weidenschilling 2002; Ford et al. 2003). The majority of planets orbiting beyond $1 \mathrm{AU}$ and in eccentric orbits currently have no other giant planet orbiting the star within 5 AU. This absence of another planet may be due to its having been ejected long ago or to the wide orbit in which it now resides, giving no discernible Doppler signal.

Alternatively, the common lone planets in eccentric orbits add import to theories that explain the origin of the eccentricity without need of any additional giant planets. Compelling models include those that invoke perturbations by the protoplanetary disk to pump eccentricities (Goldreich \& Sari 2003), as no other planet is required. Further, theories that invoke mutual perturbations among a multitude of planetesimals are also intriguing, as such dynamics may be common (Levison et al. 1998). Further observations of single planets in eccentric orbits, combined with more sophisticated models, may help determine how frequently these various scenarios play roles in generating the eccentricities of giant planets. Interestingly, two sub-Saturn candidates exhibit nonzero eccentricity, namely, HD $16141(P=$ 75 days, $e=0.18)$ and HD $3651(P=62.2$ days, $e=0.64)$, indicating that the mechanism that pumps eccentricities continues to act for such low masses. At the other end of the mass distribution are planets so massive, such as 70 Vir b $(M \sin i=$ $7 M_{\text {Jup }}$ ), that only a similarly massive second planet could induce the large eccentricities. Indeed, for both 70 Vir no additional supermassive planets are found within $5 \mathrm{AU}$.

Remarkably, among the five planets presented here, all five have metallicities well above solar, with $[\mathrm{Fe} / \mathrm{H}]>+0.27$ (Valenti \& Fischer 2005; Fischer \& Valenti 2005). Two of the target stars were selected by virtue of their high metallicity. The planets around these two, and the apparent high metallicity of the other three stars, further strengthens the already strong suggestion that metal-rich stars have a high occurrence rate of giant planets within 3 AU (Fischer \& Valenti 2005; Santos et al. 2004a), reaching $\sim 20 \%$ for $[\mathrm{Fe} / \mathrm{H}]>+0.3$.

We thank A. Cumming, J. Scargle, E. Ford, and P. Stark for valuable discussions about false alarm probability. We gratefully acknowledge the efforts and dedication of the Keck Observatory staff. We appreciate support by NASA grant NAG5-75005 and by NSF grant AST 03-07493 (to S. S. V.); also support by NSF grant AST 99-88087, by NASA grant NAG5-12182, and travel support from the Carnegie Institution of Washington (to R. P. B.). G. W. H. acknowledges support from NASA grant NCC5-511 and NSF grant HRD-9706268. We are also grateful for support by Sun Microsystems. We thank the NASA and UC Telescope assignment committees for allocations of telescope time toward the planet search around $\mathrm{M}$ dwarfs. This research has made use of the Simbad database, operated at CDS, Strasbourg, France. The authors wish to extend special thanks to those of Hawaiian ancestry on whose sacred mountain of Mauna Kea we are privileged to be guests. Without their generous hospitality, the Keck observations presented herein would not have been possible.

\section{REFERENCES}

Allende Prieto, C., \& Lambert, D. L. 1999, VizieR Online Data Catalog, 335, 20555

Armitage, P. J., Livio, M., Lubow, S. H., \& Pringle, J. E. 2002, MNRAS, 334, 248

Baliunas, S. L., et al. 1995, ApJ, 438, 269

Bevington, P. R., \& Robinson, D. 1992, Data Reduction and Error Analysis for the Physical Sciences (2nd ed.; New York: McGraw-Hill)

Bodenheimer, P., Laughlin, G., \& Lin, D. N. C. 2003, ApJ, 592, 555

Butler, R. P., Marcy, G. W., Vogt, S. S., Fischer, D. A., Henry, G. W., Laughlin, G., \& Wright, J. T. 2003, ApJ, 582, 455

Butler, R. P., Marcy, G. W., Williams, E., McCarthy, C., Dosanjh, P., \& Vogt, S. S. 1996, PASP, 108, 500

Butler, R. P., Vogt, S. S., Marcy, G. W., Fischer, D. A., Wright, J. T., Henry, G. W., Laughlin, G., \& Lissauer, J. J. 2004, ApJ, 617, 580

Butler, R. P., et al. 2002, ApJ, 578, 565

Carter, B. D., Butler, R. P., Tinney, C. G., Jones, H. R. A., Marcy, G. W.,

McCarthy, C., Fischer, D. A., \& Penny, A. J. 2003, ApJ, 593, L43

Chiang, E. I., \& Murray, N. 2002, ApJ, 576, 473

Cochran, W. D., Hatzes, A. P., Butler, R. P., \& Marcy, G. W. 1997, ApJ, 483, 457

Cumming, A., Marcy, G. W., \& Butler, R. P. 1999, ApJ, 526, 890

Cumming, A., Marcy, G. W., Butler, R. P., \& Vogt, S. S. 2003, in ASP Conf. Ser. 294, Scientific Frontiers in Research on Extrasolar Planets, ed. D. Deming \& S. Seager (San Francisco: ASP), 27

Eaton, J. A., Henry, G. W., \& Fekel, F. C. 2003, in The Future of Small Telescopes in the New Millennium, Vol. II, ed. T. D. Oswalt (Dordrecht: Kluwer), 189

ESA. 1997, VizieR Online Data Catalog, 1239, 0

Feltzing, S., \& Gustafsson, B. 1998, A\&AS, 129, 237

Fischer, D. A., Butler, R. P., Marcy, G. W., Vogt, S. S., \& Henry, G. W. 2003a, ApJ, 590, 1081

Fischer, D. A., \& Valenti, J. A. 2005, ApJ, in press

Fischer, D. A., et al. 2003b, ApJ, 586, 1394

Ford, E. B. 2005, AJ, submitted (astro-ph/0305441)

Ford, E. B., Rasio, F. A., \& Yu, K. 2003, in ASP Conf. Ser. 294, Scientific Frontiers in Research on Extrasolar Planets, ed. D. Deming \& S. Seager (San Francisco: ASP), 181

Gilliland, R. L., \& Baliunas, S. L. 1987, ApJ, 314, 766

Girardi, L., Bertelli, G., Bressan, A., Chiosi, C., Groenewegen, M. A. T., Marigo, P., Salasnich, B., \& Weiss, A. 2002, A\&A, 391, 195

Goldreich, P., \& Sari, R. 2003, ApJ, 585, 1024

Henry, G. W. 1995a, in ASP Conf. Ser. 79, Robotic Telescopes: Current Capabilities, Present Developments, and Future Prospects for Automated Astronomy, ed. G. W. Henry \& J. A. Eaton (San Francisco: ASP), 44

. 1995b, in ASP Conf. Ser. 79, Robotic Telescopes: Current Capabilities, Present Developments, and Future Prospects for Automated Astronomy, ed. G. W. Henry \& J. A. Eaton (San Francisco: ASP), 37 1999, PASP, 111, 845

Henry, G. W., Baliunas, S. L., Donahue, R. A., Fekel, F. C., \& Soon, W. 2000a, ApJ, 531, 415

Henry, G. W., Marcy, G. W., Butler, R. P., \& Vogt, S. S. 2000b, ApJ, 529, L41 Henry, T. J., Soderblom, D. R., Donahue, R. A., \& Baliunas, S. L. 1996, AJ, 111,439

Horne, J. H., \& Baliunas, S. L. 1986, ApJ, 302, 757

Ida, S., \& Lin, D. N. C. 2004, ApJ, 604, 388

Jones, H. R. A., Butler, R. P., Tinney, C. G., Marcy, G. W., McCarthy, C., Penny, A. J., \& Carter, B. D. 2005, in ASP Conf. Ser. 321, Extrasolar Planets: Today and Tomorrow, ed. J. P. Beaulieu, A. Lecavelier des Etangs, \& C. Terquem (San Francisco: ASP), in press

Jones, H. R. A., Butler, R. P., Tinney, C. G., Marcy, G. W., Penny, A. J., McCarthy, C., \& Carter, B. D. 2003, MNRAS, 341, 948

Jorissen, A., Mayor, M., \& Udry, S. 2001, A\&A, 379, 992

Laughlin, G. 2000, ApJ, 545, 1064

Laws, C., Gonzalez, G., Walker, K. M., Tyagi, S., Dodsworth, J., Snider, K., \& Suntzeff, N. B. 2003, AJ, 125, 2664 
Levison, H. F., Lissauer, J. J., \& Duncan, M. J. 1998, AJ, 116, 1998 Loredo, T. J., \& Chernoff, D. F. 2003, in Statistical Challenges in Astronomy, ed. E. D. Feigelson \& G. J. Babu (New York: Springer), 57

Marcy, G. W., \& Butler, R. P. 1992, PASP, 104, 270 2000, PASP, 112, 137

Marcy, G. W., Butler, R. P., Fischer, D. A., Laughlin, G., Vogt, S. S., Henry, G. W., \& Pourbaix, D. 2002, ApJ, 581, 1375

Marcy, G. W., Butler, R. P., Fischer, D. A., \& Vogt, S. S. 2005, in ASP Conf. Ser. 321, Extrasolar Planets: Today and Tomorrow, ed. J. P. Beaulieu, A. Lecavelier des Etangs, \& C. Terquem (San Francisco: ASP), in press

Marcy, G. W., Butler, R. P., \& Vogt, S. S. 2000, ApJ, 536, L43

Marzari, F., \& Weidenschilling, S. J. 2002, Icarus, 156, 570

Mayor, M., \& Santos, N. C. 2003, in Proc. ESO-CERN-ESA Symp., Astronomy, Cosmology, and Fundamental Physics, ed. P. A. Shaver, L. DiLella, \& A. Giménez (Berlin: Springer), 359

Mayor, M., Udry, S., Naef, D., Pepe, F., Queloz, D., Santos, N. C., \& Burnet, M. 2004, A\&A, 415, 391

McArthur, B., et al. 2004, ApJ, 614, L81

McCarthy, C., Butler, R. P., Tinney, C. G., Jones, H. R. A., Marcy, G. W., Carter, B., Penny, A., \& Fischer, D. 2004, ApJ, 617, 575

Nidever, D. L., Marcy, G. W., Butler, R. P., Fischer, D. A., \& Vogt, S. S. 2002, ApJS, 141, 503

Noyes, R. W., Hartmann, L. W., Baliunas, S. L., Duncan, D. K., \& Vaughan, A. H. 1984, ApJ, 279, 763

Pace, G., \& Pasquini, L. 2004, A\&A, 426, 1021

Paulson, D. B., Saar, S. H., Cochran, W. D., \& Henry, G. W. 2004, AJ, 127, 1644
Pepe, F., Mayor, M., Galland, F., Naef, D., Queloz, D., Santos, N. C., Udry, S., \& Burnet, M. 2002, A\&A, 388, 632

Queloz, D., et al. 2001, A\&A, 379, 279

Reid, I. N. 2002, PASP, 114, 306

Rutten, R. G. M. 1984, A\&A, 130, 353

Saar, S. H., Butler, R. P., \& Marcy, G. W. 1998, ApJ, 498, L153

Santos, N. C., Israelian, G., \& Mayor, M. 2001, A\&A, 373, 1019 2004a, A\&A, 415, 1153

Santos, N. C., Israelian, G., Mayor, M., Rebolo, R., \& Udry, S. 2003, VizieR Online Data Catalog, 339, 80363

Santos, N. C., Mayor, M., Naef, D., Pepe, F., Queloz, D., Udry, S., \& Blecha, A. 2000, A\&A, 361, 265

Santos, N. C., et al. 2004b, A\&A, 426, L19

Scargle, J. D. 1982, ApJ, 263, 835

Seagroves, S., Harker, J., Laughlin, G., Lacy, J., \& Castellano, T. 2003, PASP, 115,1355

Tinney, C. G., Butler, R. P., Marcy, G. W., Jones, H. R. A., Penny, A. J., McCarthy, C., Carter, B. D., \& Bond, J. 2003, ApJ, 587, 423

Trilling, D. E., Lunine, J. I., \& Benz, W. 2002, A\&A, 394, 241

Udry, S., Mayor, M., \& Santos, N. C. 2003, A\&A, 407, 369

Valenti, J. A., \& Fischer, D. A. 2005, ApJ, in press

Vogt, S. S., Butler, R. P., Marcy, G. W., Fischer, D. A., Pourbaix, D., Apps, K., \& Laughlin, G. 2002, ApJ, 568, 352

Vogt, S. S., et al. 1994, Proc. SPIE, 2198, 362

Wright, J. T. 2004, AJ, 128, 1273

Wright, J. T., Marcy, G. W., Butler, R. P., \& Vogt, S. S. 2004, ApJS, 152, 261 